See discussions, stats, and author profiles for this publication at: https://www.researchgate.net/publication/351449879

\title{
Fractures in Low-Permeability Rocks: Can Poroelastic Effects Associated With Damage Zones Enhance Their Seismic Visibility?
}

Preprint in Journal of Geophysical Research Solid Earth · May 2021 DOI: 10.1029/2020JB021155

\section{CITATIONS}

5 authors, including:

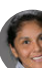

Edith Sotelo

University of Lausanne

10 PUBLICATIONS 92 CITATIONS

SEE PROFILE

Santiago G. Solazzi

University of Lausanne

14 PUBLICATIONS 40 CITATIONS

SEE PROFILE
READS

81

- J. Germán Rubino

Centro Atómico Bariloche

89 PUBLICATIONS 1,319 CITATIONS

SEE PROFILE

Nicolas Daniel Barbosa

University of Lausanne

36 PUBLICATIONS 236 CITATIONS

SEE PROFILE

Some of the authors of this publication are also working on these related projects:

Modeling seismic attenuation due to wave-induced fluid flow in partially saturated media View project

Bayesian Sequential Simulation (BSS) View project 


\title{
Fractures in low-permeability rocks: can poroelastic effects associated with damage zones enhance their seismic visibility?
}

\author{
Edith Sotelo $^{1}$, J. Germn Rubino ${ }^{2}$, Santiago G. Solazzi ${ }^{1}$, Nicols D. Barbosa ${ }^{1,3}$, \\ Klaus Holliger ${ }^{1}$ \\ ${ }^{1}$ Institute of Earth Sciences, University of Lausanne, Lausanne, Switzerland \\ ${ }^{2}$ CONICET, Centro Atmico Bariloche - CNEA, San Carlos de Bariloche, Argentina \\ ${ }^{3}$ Department of Earth Sciences, University of Geneva, Geneva, Switzerland
}

\section{Key Points:}

- We explore poroelastic effects associated with damage zones on the seismic response of fractures in largely impermeable environments.

- Our results show that such poroelastic effects can increase fracture normal compliance and reflectivity in the seismic frequency band.

- Accounting for the presence of a DZ can improve the interpretation of seismic reflectivity of fractures in this kind of environments.

Corresponding author: Edith Sotelo, edith.sotelogamboa@unil.ch

This article has been accepted for publication and ${ }^{-1}$ undergone full peer review but has not been through the copyediting, typesetting, pagination and proofreading process, which may lead to differences between this version and the Version of Record. Please cite this article as doi: 10.1029/2020JB021155.

This article is protected by copyright. All rights reserved. 


\begin{abstract}
Fluid pressure diffusion (FPD) between a fracture and a porous permeable background can increase the normal compliance of the fracture and, thus, its reflectivity. However, many fractured environments of interest are associated with background rocks that can be regarded as largely impermeable for the the typical frequencies employed in seismic surveys. Nonetheless, there is evidence to suggest that the seemingly ubiquitous presence of damaged zones (DZ) associated with fractures may provide the necessary hydraulic communication between fractures and their immediate surroundings for FPD to occur. Here, we assess the pertinence of this phenomenon. To this end, we consider a $1 \mathrm{D}$ elasticporoelastic model, which comprises a poroelastic system consisting of a fracture embedded in adjacent DZ layers. This system is enclosed in an impermeable background represented by two elastic half-spaces. We calculate the frequency-dependent $\mathrm{P}$-wave reflectivity at normal incidence at the background-DZ interface for different permeabilities, thicknesses, and porosities of the DZ. We also evaluate the corresponding normal fracture compliance. Our results show that, when accounting for the presence of a DZ surrounding an individual fracture, FPD effects between these regions induce a higher seismic reflectivity and a higher normal compliance compared to that of a hydraulically isolated fracture. This, in turn, implies that, even in largely impermeable environments, the seismic visibility of fractures can be enhanced through FPD enabled by the presence of DZ.
\end{abstract}

\title{
1 Introduction
}

Fractures are ubiquitous in geological formations and they tend to dominate their mechanical and hydraulic properties (e.g., Liu, 2005; Jaeger et al., 2009). Thus, the characterization of fractures is of great interest for wide a variety of applications such as in geothermal energy extraction, (e.g., Vidal \& Genter, 2018), $\mathrm{CO}_{2}$ storage (e.g., Ogata et al., 2014), ground water production (e.g., Ofterdinger et al., 2019), oil and gas exploitation (e.g., Gale \& Holder, 2010), nuclear waste storage (e.g., Braester, 1999), among others. Reflection seismology is a widely used, non-invasive technique for fracture detection and characterization. The basis for the application of this technique for this purpose is generally the higher compliance of fractures compared to their embedding background, which causes part of the seismic field to be reflected (e.g., Pyrak-Nolte et al., 1990; Gu et al., 1996). Classical methods to characterize fractures using seismic reflectivity are largely based on the assumption of elasticity. For instance, the characterization of fractured en- 
vironments is performed by analyzing the variation of reflectivity with angle and azimuth (e.g., Rüger, 1998; Bakulin et al., 2000; Fang et al., 2017). Similarly, some techniques to characterize isolated fractures are based on the interpretation of multiple reflections coming from the fracture surface (e.g., Minato \& Ghose, 2013, 2016). However, the works of Nakagawa and Schoenberg (2007) and Barbosa et al. (2016), which were performed in a poroelastic framework, show that, in permeable media, the hydraulic connectivity between a fracture and its background can further enhance the seismic reflectivity of the fracture. This increase in reflectivity is a direct consequence of fluid pressure diffusion (FPD) that takes place when seismic waves induce pressure gradients due to the fracturebackground mechanical contrast (e.g., White et al., 1975; Müller et al., 2010). FPD increases the normal compliance of fractures as the stiffening pore fluid exits the fracture to equilibrate the pressure (Rubino et al., 2015; Barbosa et al., 2017), which, in turn, leads to an enhancement of fracture reflectivity. In this respect, more recent work aims to capture poroelastic effects in the reflectivity analysis by considering equivalent viscoelastic models of fractured porous rock (Yang et al., 2017; He et al., 2020).

However, in many fractured environments of interest, the background is largely impermeable for the typical frequencies of seismic surveys (Rubino et al., 2014) and, hence, FPD between the fractures and their embedding background cannot take place. In fact, Barbosa et al. (2016) show that a fracture-embedding background with a permeability of $10^{-6} \mathrm{D}$ already behaves as being impermeable in the seismic frequency range. In addition, laboratory measurements performed in crystalline background rock around fault zones report permeabilities of order of $10^{-7} \mathrm{D}$ or less (Wibberley \& Shimamoto, 2003; Mitchell \& Faulkner, 2012). Nonetheless, the likely presence of damage zones (DZ) surrounding fractures can provide adequate conditions for FPD to prevail. Indeed, there is far-reaching evidence indicating the ubiquitous presence of DZ surrounding fractures and faults. In this regard, Kim et al. (2004) presents a detailed description of DZ associated with faults. They define a DZ as the volume of deformed rock resulting from the different interactions associated with the slip along faults. They further describe the DZ as being comprised of different auxiliary fractures and faults, classifying them according to their position along a fault. Several other studies show evidence of the existence of macro- and micro- fractures within the DZ, generally with decaying density as the distance from the fault core increases (Mitchell \& Faulkner, 2009; Faulkner et al., 2011; Savage \& Brodsky, 2011). The existence of DZ has also been related to naturally occurring hydraulic fracturing. For example, there is evidence of rock deformation that includes 
brecciation and focalized fracturing that accompanies the formation of magma driven dikes (Delaney \& Pollard, 1981; Brown et al., 2007). Similarly, Engvik et al. (2005) report the presence of alteration zones comprised of healed micro-cracks surrounding veins. Furthermore, studies show that there is an increase of permeability in the DZ associated to the presence of secondary fractures (Mitchell \& Faulkner, 2012) and breccias (Sruoga et al., 2004; Sruoga \& Rubinstein, 2007). In this respect, laboratory and field measurements performed on DZ and fault zones report enhancements of permeability up to $10^{-2}$ D (Brace, 1984; Wibberley \& Shimamoto, 2003). Although, the existence of healed cracks is not likely to increase permeability, stimulation treatments, such as hydraulic fracturing, which are commonly used in geothermal and hydrocarbon applications, have the potential to re-activate such sealed pre-existing fractures (Gale \& Holder, 2010; Dahi Taleghani et al., 2013). Thus, it is very likely that the existence of a DZ allows hydraulic communication with the associated fracture and, hence, promotes FPD between these regions for the frequencies typically employed in seismic surveys. However, the likely influence of the presence of a permeable $\mathrm{DZ}$ on fracture reflectivity remains so far largely unexplored.

In this work, we investigate the effects of FPD on the reflectivity and normal compliance of an isolated fracture in the presence of a DZ within an otherwise impermeable background. To capture FPD effects, we consider an elastic-poroelastic model that comprises two poroelastic DZ layers embedding a poroelastic fracture. For comparison, we also consider the purely elastic model with the same media configuration. In both models, elastic half-spaces represent the surrounding impermeable background. We calculate $\mathrm{P}$-wave reflectivities at normal incidence at the background-DZ interface for the respective models for various frequencies, DZ permeabilities, thicknesses and porosities. We also calculate the corresponding normal fracture compliance values. Moreover, we study the effects of a range of pertinent rock and fluid properties on FPD and reflectivity.

\section{Theory and methods}

\subsection{D models and governing equations}

To analyze FPD effects between an isolated fracture and its associated DZ, we consider a 1D elastic-poroelastic model (Figure 1). In this model, the thin poroelastic layer $\Lambda_{3}$ represents the fracture and its embedding poroelastic layers $\Lambda_{2}$ and $\Lambda_{4}$ the associated DZ. This poroelastic DZ-fracture set is enclosed by two elastic half-spaces $\Lambda_{1}$ and 
$\Lambda_{5}$ that represent the background rock, which is assumed to be elastic and impermeable. Additionally, we denote as $\Pi_{1}$ and $\Pi_{4}$ the respective interfaces between the elastic background and poroelastic DZ layers and as $\Pi_{2}$ and $\Pi_{3}$ the interfaces between the poroelastic fracture and DZ layers. For comparison, we also consider a purely elastic model presenting the same configuration as the elastic-poroelastic one. In the methodology and results sections, we shall illustrate that, at high-enough frequencies, the reflectivity of the elasticporolastic model converges to that of the purely-elastic one.

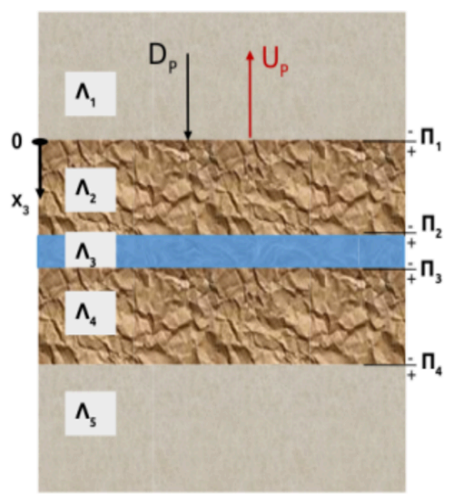

Figure 1. Layered model considered for both the elastic-poroelastic and purely elastic analyses. $D_{P}$ and $U_{P}$ are the downgoing and upgoing P-waves, respectively. $\Lambda_{1}$ and $\Lambda_{5}$ are half-spaces representing the elastic impermeable background; $\Lambda_{3}$ is the thin layer representing the fracture, and $\Lambda_{2}$ and $\Lambda_{4}$ are the layers representing the DZ. For the elastic-poroelastic case, the fracture and DZ layers are poroelastic, while for the purely elastic case, all media are elastic.

We assume a normally incident P-wave striking at the background-DZ interface $\Pi_{1}$. Then, our objective is to find the corresponding PP reflection coefficients $R_{P P}$ for both the elastic-poroelastic and purely elastic case, respectively. We compute the corresponding reflection coefficients at the DZ-background interface because it quantifies the amplitude of the reflected signal from the DZ-fracture system that could be recorded in a seismogram. For this computation, we formulate the poroelastic and elastic wave equations in the space-frequency domain, assuming that the medium is isotropic. To formulate the poroelastic wave equation, we let $\boldsymbol{u}^{s}=\boldsymbol{u}^{s}(\boldsymbol{x}, \omega)$ and $\boldsymbol{w}=\boldsymbol{w}(\boldsymbol{x}, \omega)$ be the solid displacement vector and the relative fluid displacement vector, respectively, for any position $\boldsymbol{x}$ and angular frequency $\omega$. Moreover, we let $\boldsymbol{\sigma}$, and $p_{f}$ be the total stress tensor and pore fluid pressure, respectively, which act upon the poroelastic medium. Then, we 
express the corresponding equations of motion as (Biot, 1962):

$$
\begin{aligned}
& -\omega^{2} \rho_{b} \boldsymbol{u}^{s}-\omega^{2} \rho_{f} \boldsymbol{w}=\nabla . \boldsymbol{\sigma}, \\
& -\omega^{2} \rho_{f} \boldsymbol{u}^{s}-\omega^{2} g(\omega) \boldsymbol{w}+i \omega b(\omega) \boldsymbol{w}=-\nabla p_{f} .
\end{aligned}
$$

The constitutive equations are:

$$
\begin{aligned}
& \boldsymbol{\sigma}=\mu\left(\nabla \boldsymbol{u}^{s}+\nabla \boldsymbol{u}^{s T}\right)+\left(\lambda \nabla \cdot \boldsymbol{u}^{s}+\alpha M \nabla \cdot \boldsymbol{w}\right) \boldsymbol{I}, \\
& p_{f}=-\alpha M \nabla \cdot \boldsymbol{u}^{s}-M \nabla \cdot \boldsymbol{w}
\end{aligned}
$$

where $\rho_{b}$ and $\rho_{f}$ are the bulk density of the saturated porous medium and the density of the pore fluid, respectively, $\mu$ is the frame shear modulus, $\phi$ is the porosity, $\boldsymbol{I}$ is the identity matrix, $i$ is the imaginary unit, $\lambda$ is the undrained Lam modulus, $\alpha$ is the BiotWillis effective stress coefficient, $M$ is the Biot's fluid storage modulus, and $g(\omega)$ and $b(\omega)$ are the mass coupling and viscous coefficients, respectively. The required rock physical properties are calculated as follows (e.g., Barbosa et al., 2016):

$$
\begin{aligned}
& \rho_{b}=(1-\phi) \rho_{s}+\phi \rho_{f}, \\
& \lambda=K_{m}-\frac{2}{3} \mu+\alpha^{2} M, \\
& \alpha=1-\frac{K_{m}}{K_{s}}, \\
& M=\left(\frac{\alpha-\phi}{K_{s}}+\frac{\phi}{K_{f}}\right)^{-1}, \\
& g(\omega)=\frac{1}{\omega} \Im\left(\frac{\eta}{\kappa_{d}(\omega)}\right), \\
& b(\omega)=\Re\left(\frac{\eta}{\kappa_{d}(\omega)}\right),
\end{aligned}
$$

where $\rho_{s}$ is the density of the solid grains, $K_{m}, K_{s}$, and $K_{f}$ are the bulk moduli of the drained solid frame, the solid grains, and the pore fluid, respectively. Additionally, $\eta$ is the viscosity of the pore fluid and $\kappa_{d}(\omega)$ is the dynamic permeability of the porous rock, which can be expressed as (Johnson et al., 1987):

$$
\kappa_{d}(\omega)=\kappa\left(\sqrt{1+\frac{4 i \omega}{n_{j} \omega_{B}}}+\frac{i \omega}{\omega_{B}}\right)^{-1} .
$$

Here, $\kappa$ is the static permeability of the porous medium, $\omega_{B}$ is Biot's angular frequency, which can be expressed as:

$$
\omega_{B}=\frac{\eta \phi}{\rho_{f} \kappa S},
$$

where $S$ is the tortuosity of the pore space. Finally, $n_{j}$ is a parameter that can be expressed as (Johnson et al., 1987):

$$
n_{j}=\frac{\phi \Lambda^{2}}{\kappa S}
$$

$$
-6-
$$


where $\Lambda$ is a parameter proportional to the pore-volume-to-surface ratio and has units of length (Johnson et al., 1987). According to numerical and experimental studies (e.g., Charlaix et al., 1988; Sheng \& Zhou, 1988; Smeulders et al., 1992), $n_{j}=8$ is a reasonable approximation for most porous media and hence, we use this value in the following. In this context, it is, however, important to remark that the prevailing range of $n_{j}$ for fractured porous media remains, as of today, unexplored. In spite of this uncertainty, it is expected that variations in $n_{j}$ will produce negligible changes on the predicted dynamic permeability $\kappa_{d}(\omega)$, since its decay is proportional to $\sqrt{2 / n_{j}}$ (Pride, 2003). Most importantly, $n_{j}$ impacts the behavior of $\kappa_{d}(\omega)$ only at sufficiently high frequencies, that is $\omega>>\omega_{B}$. As explained later in section 2.3, this work focuses on the poroelastic response at lower frequencies, that is $\omega<<\omega_{B}$, where $\kappa_{d}(\omega)$ approaches the value of the static permeability $\kappa$.

To formulate the elastic wave equation, we let $\boldsymbol{u}^{e}=\boldsymbol{u}^{e}(\boldsymbol{x}, \omega)$ be the displacement vector for any position $\boldsymbol{x}$ in the elastic medium and angular frequency $\omega$. We also let $\boldsymbol{\sigma}^{e}$ be the stress tensor field acting upon the medium. Then, we express the corresponding equations of motion as:

$$
-\rho_{b} \omega^{2} \boldsymbol{u}^{e}=\nabla \cdot \boldsymbol{\sigma}^{e}
$$

The associated constitutive equation is given by:

$$
\boldsymbol{\sigma}^{e}=\mu\left(\nabla \boldsymbol{u}^{e}+\nabla \boldsymbol{u}^{e T}\right)+\lambda \nabla \cdot \boldsymbol{u}^{e} \boldsymbol{I} .
$$

\subsection{Solution for displacements and PP reflection coefficients}

\subsubsection{Total displacements}

We assume that an incident $\mathrm{P}$-wave propagates downwards, in the $\hat{\boldsymbol{x}}_{\boldsymbol{3}}$ direction (Figure 1), and strikes the interface $\Pi_{1}$ at normal incidence. Under this condition, for the elastic-poroelastict model, the propagating modes present in the elastic half spaces $\Lambda_{1}$ and $\Lambda_{5}$ are P-waves, while in the poroelastic layers $\Lambda_{2}, \Lambda_{3}$ and $\Lambda_{4}$, both fast $\left(P_{1}\right)$ and slow $\left(P_{2}\right) \mathrm{P}$-waves are present. For the purely elastic model, only $\mathrm{P}$-waves are present throughtout the model. Note as well that the only non-zero component of the displacement vectors is in $\hat{\boldsymbol{x}}_{\boldsymbol{3}}$. Then, to find the total displacements at each medium $\Lambda_{m}$, with $m=1, . ., 5$, we sum the corresponding displacements produced by the waves traveling in the given medium $\Lambda_{m}$.

For the elastic-poroelastic model, we need to consider two types of media: elastic and poroelastic. We let $u_{n}^{\epsilon}$ be the total displacement for each elastic medium $n$, where 
$n$ refers to the upper half-space $\Lambda_{1}$ and lower half-space $\Lambda_{5}$, respectively. When $n$ corresponds to $\Lambda_{1}$ the expression for $u_{n}^{\epsilon}$ is given by:

$$
u_{n}^{\epsilon}=u_{n D_{P}}^{\epsilon}+u_{n U_{P}}^{\epsilon}
$$

where $D$ and $U$ refer to the downgoing and upgoing waves, respectively, and the subscript $P$ refers to the P-wave. Since in the lower half-space $\Lambda_{5}$ there are no upgoing-waves, $u_{n}^{\epsilon}$ simplifies to:

$$
u_{n}^{\epsilon}=u_{n D_{P}}^{\epsilon}
$$

For each poroelastic layer $d$, with $d=\Lambda_{2}, \Lambda_{3}$, and $\Lambda_{4}$, we let $u_{d}^{s}$ be the total solid displacement and $w_{d}$ the total relative fluid displacement, respectively. Then, we express these total displacements as:

$$
u_{d}^{s}=\sum_{q} u_{d q}^{s}, \quad w_{d}=\sum_{q} w_{d q},
$$

with $q=D_{P_{1}}, D_{P_{2}}, U_{P_{1}}, U_{P_{2}}$, where the subscripts $P_{1}$ and $P_{2}$ indicate the associated fast and slow $\mathrm{P}$-waves, respectively.

On the other hand, for the purely elastic model, we let $u_{n}^{e}$ be the elastic displacement component for each elastic medium $n=\Lambda_{1}, . ., \Lambda_{5}$. The expression for the total displacement $u_{n}^{e}$ for each medium $n$, except $\Lambda_{5}$, is then given by equation (9). For $\Lambda_{5}$, the total displacement $u_{n}^{e}$ is given by equation (10). We remark that the respective terms in equations (9) and (10) are replaced by $u_{n}^{e}$ and $u_{n j}^{e}$, where $j$ can be either $D_{P}$ or $U_{P}$.

\subsubsection{Solution for displacements}

For the elastic-poroelastic model, we express the corresponding solution $u_{n j}^{\epsilon}$ for each elastic medium $n=\Lambda_{1}$ and $\Lambda_{5}$, with $j=D_{P}$ or $U_{P}$, as:

$$
u_{n j}^{\epsilon}=E_{n j}^{\epsilon} \exp \left[ \pm i k_{n}^{\epsilon} x_{3}\right]
$$

where $E_{n j}^{\epsilon}$ is the amplitude of the corresponding elastic displacement and $x_{3}$ is the position. Negative and positive signs in the exponential correspond to downgoing and upgoing waves, respectively. $k_{n}^{\epsilon}$ is the elastic scalar wavenumber for the $\mathrm{P}$-wave in medium $n$, calculated as $k_{n}^{\epsilon}=\omega / V_{P}^{n}$, where $V_{P}^{n}$ is the P-wave velocity of medium $n$. The corresponding $V_{P}^{n}$ is:

$$
V_{P}^{n}=\sqrt{\frac{\lambda^{n}+2 \mu^{n}}{\rho_{b}^{n}}} .
$$

Here, $\lambda$ and $\rho_{b}$ are the undrained Lam modulus and the bulk density, respectively (equation (3)). For the poroelastic layers $d=\Lambda_{2}, \Lambda_{3}$, and $\Lambda_{4}$, we express the solution for the 
solid and relative fluid displacement $u_{d q}^{s}$ and $w_{d q}$ as:

$$
\begin{aligned}
& u_{d q}^{s}=S_{d q} \exp \left[ \pm i k_{d j} x_{3}\right], \\
& w_{d q}=W_{d q} \exp \left[ \pm i k_{d j} x_{3}\right],
\end{aligned}
$$

where $S_{d q}$ and $W_{p q}$ are the amplitudes of the solid and relative fluid displacements, respectively. Additionally, $k_{d j}$ is the poroelastic scalar wavenumber for the wave $j$ in layer $d$, with $j=P_{1}$ when $q=D_{P_{1}}, U_{P_{1}}$ and $j=P_{2}$ when $q=D_{P_{2}}, U_{P_{2}}$. Please note that the scalar wavenumber $k_{d j}$ is complex-valued, frequency-dependent and its real part is associated with the phase velocity. To obtain $k_{d j}$, we follow the procedure employed by Barbosa et al. (2016).

For the purely elastic model, the expression for the solution of $u_{n j}^{e}$ for each elastic medium $n, n=\Lambda_{1}, . ., \Lambda_{5}$, is the same as the one stated in equation (12), after replacing the corresponding terms by $E_{n j}^{e}$ and $k_{n}^{e}$.

\subsubsection{PP reflection coefficient}

We aim to find the PP reflection coefficients $R_{P P}=R_{P P}^{\epsilon}$ and $R_{P P}=R_{P P}^{e}$ at the interface $\Pi_{1}$ of the half-space $\Lambda_{1}$ in both the elastic-poroelastic and purely elastic models, respectively (Figure 1). Without loss of generality, we assume that the amplitude of the incident P-wave is one: $E_{\Lambda_{1} D_{P}}^{\epsilon}=E_{\Lambda_{1} D_{P}}^{e}=1$. Then, we seek to find the amplitudes of the reflected $\mathrm{P}$-waves at the interface $\Pi_{1}$ of the upper half-space $\Lambda_{1}: R_{P P}^{\epsilon}=E_{\Lambda_{1} U_{P}}^{\epsilon}$ and $R_{P P}^{e}=E_{\Lambda_{1} U_{P}}^{e}$. To this end, we assemble sets of linear equations, which we find by imposing suitable continuity conditions at the corresponding media interfaces.

For the elastic-poroelastic model, we distinguish two types of interfaces: elasticporoelastic and purely poroelastic ones. At the elastic-poroelastic interfaces $\Pi_{q}$, for $q=$ 1, 4, we impose continuity of solid displacements and tractions and we set to zero the relative fluid displacements, respectively (Deresiewicz \& Skalak, 1963):

$$
\begin{aligned}
& \left.\left(u_{n}^{\epsilon}-u_{d}^{s}\right)\right|_{\Pi_{q}}=0, \\
& \left.\left(t_{n}^{\epsilon}-t_{d}\right)\right|_{\Pi_{q}}=0, \\
& \left.w_{d}^{s}\right|_{\Pi_{q}}=0 .
\end{aligned}
$$

For $q=1$, the corresponding media are $n=\Lambda_{1}$ and $d=\Lambda_{2} ;$ for $q=4$, they are $n=$ $\Lambda_{5}$ and $d=\Lambda_{4}$. We calculate the traction component $t_{n}^{\epsilon}$ as $t_{n}^{\epsilon}=\left(\boldsymbol{\sigma}^{\epsilon} \cdot \hat{\boldsymbol{x}}_{\boldsymbol{3}}\right) \cdot \hat{\boldsymbol{x}}_{\boldsymbol{3}}$. Then, using equation (8) to replace $\boldsymbol{\sigma}^{\epsilon}$, we express $t_{n}^{\epsilon}$ as:

$$
t_{n}^{\epsilon}=\left(\lambda^{n}+2 \mu^{n}\right)\left(u_{n}^{\epsilon}\right)_{, 3},
$$


where ()$_{, 3}=\partial(\cdot) / \partial x_{3}$. Similarly, we calculate the traction component $t_{d}$ as $t_{d}=\left(\boldsymbol{\sigma} \cdot \hat{\boldsymbol{x}}_{\mathbf{3}}\right)^{\cdot} \hat{\boldsymbol{x}}_{\mathbf{3}}$ and, using equation (2) to replace $\boldsymbol{\sigma}$, we obtain $t_{d}$ :

$$
t_{d}=\left(\lambda^{d}+2 \mu^{d}\right)\left(u_{d}^{s}\right)_{, 3}+\alpha^{d} M^{d}\left(w_{d}\right)_{3} .
$$

At the purely poroelastic interfaces $\Pi_{q}$ with $q=2,3$, we impose the continuity of solid displacements, relative fluid displacements, tractions, and fluid pressures, respectively (Deresiewicz \& Skalak, 1963):

$$
\begin{aligned}
& \left.\left(u_{d}^{s}-u_{(d+1)}^{s}\right)\right|_{\Pi_{q}}=0, \\
& \left.\left(w_{d}-w_{(d+1)}\right)\right|_{\Pi_{q}}=0, \\
& \left.\left(t_{d}-t_{(d+1)}\right)\right|_{\Pi_{q}}=0, \\
& \left.\left(p_{f d}-p_{f(d+1)}\right)\right|_{\Pi_{q}}=0 .
\end{aligned}
$$

Here, $d=\Lambda_{q}$ and $(d+1)=\Lambda_{(q+1)}$. Moreover, we calculate $t_{d}$ and $t_{(d+1)}$ using equation (17). Additionally, using equation (2), we evaluate the pore fluid pressure:

$$
p_{f d}=-\alpha^{d} M^{d}\left(u_{d}^{s}\right)_{, 3}-M^{d}\left(w_{d}\right)_{, 3} .
$$

To complete the system of equations, we express the relative fluid displacement in terms of the solid displacement through $\gamma_{d j}=W_{d j} / S_{d j}$ where $j=P_{1}, P_{2}$. This ratio can be obtained from the properties of the porous medium (Barbosa et al., 2016).

For the elastic model, we obtain the corresponding system of equations by imposing the continuity of displacements and tractions at each medium interface $\Pi_{q}$ with $q=$ $1, . ., 4$, respectively:

$$
\begin{aligned}
& \left.\left(u_{n}^{e}-u_{(n+1)}^{e}\right)\right|_{\Pi_{q}}=0 \\
& \left.\left(t_{n}^{e}-t_{(n+1)}^{e}\right)\right|_{\Pi_{q}}=0 .
\end{aligned}
$$

Here, $n=\Lambda_{q}$ and $(n+1)=\Lambda_{(q+1)}$. We calculate $t_{n}^{e}$ and $t_{(n+1)}^{e}$ using equation (16) after replacing the corresponding displacement term by $u_{n}^{e}$.

\subsection{FPD frequency regimes}

When seismic waves propagate through heterogeneous materials, pore fluid pressure perturbations arise between regions of differing compresibilities. These pressure gradients are equilibrated through FPD, which, depending on the size of the underlying heterogeneities, prevails at different scales. Our analysis focuses on the mesoscopic scale, which refers to those heterogeneities that are larger than the pore size but much smaller 
than the wavelength of the propagating wave. For the case of compliant fractures embedded in a much stiffer DZ, the compressibility contrast allows seismic waves to induce strong fluid pressure gradients and associated fluid flow.

On the other hand, it is important to notice that FPD prevails at frequencies much lower than Biot's characteristic frequency of the medium: $f \ll f_{B}$, with $f_{B}=\omega_{B} /(2 \pi)$ (equation 5). At these sufficiently low frequencies, the fluid flow within the pores is viscousdominated, provided that the thickness of the viscous boundary layer remains greater than the characteristic pore size (Johnson et al., 1987). Under this condition, the imaginary part of the dynamic permeability $k_{d}(\omega)$ becomes negligible (equation 4$)$. Moreover, in the low-frequency limit, $k_{d}(\omega)$ becomes real-valued and frequency-independent and equal to the static permeability $\kappa: \lim _{\omega \rightarrow 0} k_{d}(\omega)=\kappa$. If we additionally constrain the analysis of Biot's equations to the quasi-static case, it can be shown that the behavior of the slow $\mathrm{P}$-wave is described by a pressure diffusion equation with diffusion coefficient $D$ (Chandler \& Johnson, 1981):

$$
D=\frac{\kappa}{\eta} \frac{M H_{d}}{H}
$$

where the drained and undrained plane-wave moduli $H_{d}$ and $H$ can be calculated as $H_{d}=$ $K_{m}+4 / 3 \mu$ and $H=\lambda+2 \mu$, respectively. Moreover, we let $L_{d}$ be the characteristic diffusion length (Norris, 1993):

$$
L_{d}=\sqrt{\frac{D}{\omega}} .
$$

As the frequency varies, distinct FPD regimes can be identified according to the relative magnitudes between the scale of a heterogeneity and its characteristic diffusion length. For the case of a fracture surrounded by DZ, the relevant scales are their respective thicknesses. For simplicity, let us assume that the thickness of the fracture $h^{c}$ is negligible compared to that of the DZ and that its diffusion coefficient (equation (21)) is very high. In this context, we have $h^{c} \ll L_{d}^{c}$ for the frequency range of interest. Here, the superscript $c$ refers to the fracture. Conversely, if we consider that the DZ thickness is much larger than that of the fracture but its permeability is much lower, then we expect that the relationship between DZ thickness $h^{z}$ and its characteristic diffusion length $L_{d}^{z}$ varies from $h^{z} \ll L_{d}^{z}$ to $h^{z} \gg L_{d}^{z}$ as frequency increases. Thus, for the fracture-DZ poroelastic system, we can regard the thickness of the DZ $h^{z}$ as the relevant mesoscopic heterogeneity scale controlling FPD. Under this perspective, we distinguish the following two endmember regimes for FPD: relaxed and unrelaxed. The relaxed state occurs at sufficiently low frequencies, at which the diffusion length $L_{d}^{z}$ is larger than the thickness of the DZ $h^{z}$. Thus, there is enough time for the pressure between the fracture and DZ layers to 
equilibrate. Conversely, the unrelaxed state occurs at sufficiently high frequencies, at which the diffusion length $L_{d}^{z}$ is very small compared to thickness of the DZ $h^{z}$ and, consequently, there is no time for FPD to take place, and the medium behaves as hydraullically isolated. A transition zone exists at intermediate frequencies, at which the diffusion lengths are of comparable size to that of the thickness of the $\mathrm{DZ} h^{z}$. This zone is characterized by a transition frequency $f_{c}=\omega_{c} / 2 \pi$, which can be estimated as (Brajanovski et al., 2006; Müller \& Rothert, 2006):

$$
w_{c} \approx \frac{9}{2} \frac{D^{z}}{\left(h^{z}\right)^{2}}
$$

We remark that Brajanovski et al. (2006) and Müller and Rothert (2006) have also pointed to the existence of a second characteristic frequency that, depending on the DZ and fracture properties, could be visible in the transition zone. However, for the rock and fluid properties we are using in this work, this second characteristic frequency is not visible.

In this work, we consider an open fracture whose permeability is several orders of magnitude greater than the DZ. Then, it is expected that $f_{B}^{c}<<f_{B}^{z}$, meaning that FPD within the fracture is limited to much lower frequencies than for the DZ. Particularly, for frequencies greater than $f_{B}^{c}$ but lower than $f_{B}^{z}$, FPD does no longer take place within the fracture since fluid flow becomes inertial-dominated, but FPD is still present within the DZ. We remark that, the proposed solutions for amplitude displacements, as expressed in equation (12), account for both fluid flow regimes, viscous- and inertial-dominated, since they include the dynamic permeability (equation 4) in the calculation of the poroelastic wavenumbers of the fracture and DZ. Therefore, within this frequency band, pressure equilibration will take place under two different flow regimes. Nonetheless, due to the greater thickness and lower permeability of the DZ, it is expected that the viscousdominated fluid flow regime in this region controls the reflectivity response of the DZfracture system. Moreover, hereinafter we use the terms low- and high-frequency limits within the FPD context. Meaning that, they signify the relaxed and unrelaxed FPD regimes, respectively.

\subsection{Normal fracture compliance}

Fracture compliance defines the mechanical behavior of a fracture. The more compliant a fracture is, the easier it undergoes deformation and the higher is its seismic reflectivity since the mechanical contrast with the background increases. For the case of FPD effects caused by a normally incident P-wave, our interest focuses on normal fracture compliance. For the fracture-DZ poroelastic system, FPD allows fluid to flow from 
the more compliant fracture to the stiffer DZ during half of a wave cycle, which, in turn, decreases the stiffening effect of the fracture fluid, thus increasing the normal compliance of the fracture and its reflectivity. However, the extent to which normal fracture compliance and its reflectivity increase is controlled by the FPD regimes. Normal compliance is maximal, associated with a maximal increase of reflectivity, when FPD is in its relaxed state, that is, when the fracture fluid is allowed to exit until the pressure fully equilibrates. In contrast, normal fracture compliance is lowest, with no reflectivity enhancement during the unrelaxed FPD regime, in which the fracture behaves as hydraulically isolated. Intermediate values of normal fracture compliance are expected as FPD transitions from its relaxed to its unrelaxed regime.

We calculate the normal fracture compliance $Z_{N}^{e}$ for the elastic fracture represented as a thin layer using the definition introduced by Schoenberg (1980). Then, extending this concept to a poroelastic framework in a similar way to Rubino et al. (2015), we also calculate the normal fracture compliance $Z_{N}^{p}$ for the poroelastic fracture also represented as a thin layer:

$$
\begin{aligned}
Z_{N}^{e}=\frac{\left.u_{n}^{e}\right|_{\Pi_{3}}-\left.u_{n}^{e}\right|_{\Pi_{2}}}{\bar{t}_{n}^{e}}, \quad \text { with } \quad \bar{t}_{n}^{e}=\frac{\left.t_{n}^{e}\right|_{\Pi_{2}}+\left.t_{n}^{e}\right|_{\Pi_{3}}}{2}, \\
Z_{N}^{p}=\frac{\left.u_{d}^{s}\right|_{\Pi_{3}}-\left.u_{d}^{s}\right|_{\Pi_{2}}}{\bar{t}_{d}}, \quad \text { with } \quad \bar{t}_{d}=\frac{\left.t_{d}\right|_{\Pi_{2}}+\left.t_{d}\right|_{\Pi_{3}}}{2},
\end{aligned}
$$

with $n=d=\Lambda_{3}$. Here, we do not imply that it is seismically equivalent to represent the 1D poroelastic DZ-fracture system by a slip interface characterized by a poroelastic normal compliance equal to $Z_{N}^{p}$. But the main purpose of calculating $Z_{N}^{p}$ is to show the effect of FPD on normal fracture compliance. We refer the reader to the first paragraph of the discussion section for further details.

On the other hand, we express the normal fracture compliance in the relaxed and unrelaxed FPD regimes, $Z_{N}^{o}$ and $Z_{N}^{u}$, as (Rubino et al., 2015):

$$
\begin{aligned}
& Z_{N}^{o}=Z_{N}^{u}+\frac{2 B^{c}\left(B^{c}-B^{z}\right)}{\frac{2 B^{c}}{\alpha^{z} Z_{N}^{d}}+\frac{M^{z}\left(1-\alpha^{z} B^{z}\right)}{h^{z}}}, \\
& Z_{N}^{u}=\frac{h^{c}}{H^{c}} .
\end{aligned}
$$

Here, $B$ is the Skempton coefficient, which can be written as $B=\alpha M / H$. Note that, $Z_{N}^{o}$ and $Z_{N}^{u}$ can also be designated as the low- and high-frequency limits of fracture compliance, respectively. In the context of FPD, $Z_{N}^{o}$ and $Z_{N}^{u}$ are the maximum and minimum values that the normal compliance of a fracture can assume for a given set of rock and fluid properties. In particular, the high frequency-limit of normal fracture compliance $Z_{N}^{u}$ corresponds to the elastic behavior of the fracture since at sufficiently high 
frequencies there is no time for FPD to take place and the fracture behaves as hydraulically isolated. This, in turn, impedes the outflow of the stiffening fluid from the fracture causing its compliance to decrease to this minimum value. Moreover, as detailed by equation 25 , the high-frequency limit of fracture compliance $Z_{N}^{u}$ only depends on the fracture physical properties. In this work, we use the ratio $Z_{N}^{o} / Z_{N}^{u}$ as a measure of the maximum increase of normal fracture compliance due to FPD with respect to its elastic limit. We remark that Rubino et al. (2015) find the expressions for $Z_{N}^{o}$ and $Z_{N}^{u}$ by considering a 1D periodic system consisting of a relatively thick horizontal layer alternating with a thinner layer representing a fracture. They assume a representative elementary volume (REV) comprised of the fracture layer as well as the two embedding layers with half of their thicknesses. They also assume a no-flow condition at the upper and lower boundaries of the REV, which holds for the entire system given the symmetry of the problem and its infinite nature. For the fracture-DZ poroelastic system enclosed within elastic half-spaces considered in this work, periodicity is no longer required to ensure the no flow condition since this is, in fact imposed, by the zero relative fluid displacement boundary condition at the the interfaces between the poroelastic DZ and elastic half-spaces representing the impermeable background (equation 15). Thus, the expressions in equation (25) are applicable for our problem when we consider the entire thickness of the DZ.

\section{Results}

In this section, we present results of frequency-dependent reflectivity and normal fracture compliance for the elastic-poroelastic and elastic models. We analyze the effects of variations of rock properties of the DZ and of the fracture, as well as of the pore fluid, on the reflectivity and on the normal compliance. We remark that for high-enough frequencies, the results from the elastic-poroelastic models should converge to those obtained from the corresponding elastic models. This convergence is expected because in the highfrequency limit the unrelaxed FPD regime prevails. This effect, as previously explained, prevents fluid exchange between the poroelastic fracture and the DZ and, which as a consequence, causes them to behave elastically. For these examples, we use rock and fluid properties from Table 1, which shows the reference values of the rock and fluid properties for the poroelastic thin layer representing the fracture and the associated DZ layers. Most of these values are adopted from Barbosa et al. (2016) and Barbosa et al. (2019), with rock properties emulating those of a crystalline lithology. Fracture bulk and shear moduli, $K_{m}^{c}$ and $\mu^{c}$, are estimated using the formulae proposed by Nakagawa and Schoen- 
berg (2007):

$$
Z_{T}^{d}=h^{c} / \mu^{c} ; \quad Z_{N}^{d}=h^{c} /\left(K_{m}^{c}+4 / 3 \mu^{c}\right) .
$$

For the drained tangential $Z_{T}^{d}$ and normal $Z_{N}^{d}$ compliances, we assume values of $5 \times 10^{-10}$ $\mathrm{m} / \mathrm{Pa}$ and $1.5 \times 10^{-10} \mathrm{~m} / \mathrm{Pa}$, respectively. The magnitude of these values $\left(\sim 10^{-10} \mathrm{~m} / \mathrm{Pa}\right)$ corresponds to a fracture of around a hundred meters long (Hobday \& Worthington, 2012). For the elastic media, comprised by the elastic fracture, DZ and background, we compute the corresponding elastic moduli using Gassmann's equations (Gassmann, 1951) and we take the required rock and fluid properties from Table 1. For calculations corresponding to the elastic background and DZ, we take the necessary rock properties from the ones listed for the $\mathrm{DZ}$ and, in a similar way, for the elastic fracture, we take the required properties from the poroelastic fracture. We indicate that, for the rock and fluid properties listed in Table 1, Biot's frequencies for the poroelastic DZ and the fracture are $8.1 \times 10^{3} \mathrm{~Hz}$ and $1.2 \times 10^{3} \mathrm{~Hz}$, respectively.

Table 1. Reference values of the physical properties for the DZ, fracture, and pore fluid.

\begin{tabular}{|l|c|c|}
\hline Property & $\mathrm{DZ}$ & Fracture \\
\hline Grain bulk modulus $K_{s}(\mathrm{GPa})$ & 37 & 37 \\
Grain density $\rho_{s}\left(\mathrm{Kg} / \mathrm{m}^{3}\right)$ & 2730 & 2730 \\
Porosity $\phi$ & 0.015 & 0.8 \\
Frame bulk modulus $K_{m}(\mathrm{GPa})$ & 33 & 0.004 \\
Frame shear modulus $\mu(\mathrm{GPa})$ & 29 & 0.002 \\
Thickness $h(\mathrm{~m})$ & 0.2 & 0.001 \\
Permeability $\kappa(\mathrm{D})$ & 0.1 & 100 \\
Tortuosity $S$ & 3 & 1 \\
Fluid density $\rho_{f}\left(\mathrm{Kg} / \mathrm{m}^{3}\right)$ & 1000 & 1000 \\
Fluid bulk modulus $K_{f}(\mathrm{GPa})$ & 2.25 & 2.25 \\
Fluid viscosity $\eta(\mathrm{Pa} . \mathrm{s})$ & 0.001 & 0.001 \\
\hline
\end{tabular}

Notice that, unless stated otherwise, we use the same rock physical properties for the DZ and the background, except for the permeability, to simplify the interpretation of results, since we want to emphasize the FPD effects induced by the presence of the DZ surrounding a fracture. Furthermore, please note that we do not include intrinsic attenuation effects in the DZ, although they are expected to take place due to the pres- 
ence of macro- and micro-fractures. Nonetheless, we consider that these simplifications are justified since they aim to highlight FPD effects on the reflectivity response.

\subsection{Effect of permeability of the DZ}

In the following example, we show the effect of different DZ permeabilities on reflectivity and normal fracture compliance. As previously outlined, it is the permeability of the DZ that allows for the hydraulic communication with the adjacent fracture for FPD to take place.

Figure 2 shows the absolute value of the normal-incidence reflection coefficient $\left|R_{P P}\right|$ versus frequency for the elastic and the elastic-poroelastic models considering different $\mathrm{DZ}$ permeabilities $\kappa^{z}$. These results show that there is a maximum increase of reflectivity for the elastic-poroelastic models of approximately one order-of magnitude when compared to the elastic results for frequencies lower than the respective transition frequencies $f_{c}$. This is a consequence of FPD prevailing between the DZ and the fracture, which allows for fluid release from the fracture as the pressure equilibrates during a half wave cycle. We observe that the role of the DZ permeability $\kappa^{z}$ is to control the transition frequency, at which reflectivity decreases towards its undrained values. Here, higher permeabilities shift this transition frequency towards higher values. This is expected given that the characteristic transition frequency $f_{c}$ is directly proportional to the permeability $\kappa^{z}$ (equations 21 and 23). We also note that, for all elastic-poroelastic models, there is an upper limit for $\left|R_{P P}\right|$ regardless of the permeability $\kappa^{z}$. This is due to the fact that, irrespective of its permeability, the DZ provides a limited pore volume for FPD to occur in its relaxed state. We present a detailed analysis regarding this subject in the next subsection. Notice as well the presence of reverberations of $\left|R_{P P}\right|$ at high frequencies for a permeability of $1 \mathrm{D}$. For this permeability, the corresponding Biot's frequency in the $\mathrm{DZ}$ is $\sim 800 \mathrm{~Hz}$ and at this frequency $P_{2}$ becomes a propagating wave. Then, multiples are expected within the poroelastic DZ layer when the wavelength of $P_{2}$ becomes smaller than the layer thickness. These multiples convert to upgoing $P_{1}$-waves at the backgroundDZ interface and interfere constructively and destructively with the reflected $P_{1}$ at this interface. Furthermore, at frequencies comparable to or larger than Biot's frequency, the relaxation mechanism is no longer controlled by viscous diffusion but by inertial forces. In that case, equations (21) to (23), which assume a pressure diffusion mechanism, no longer apply. 


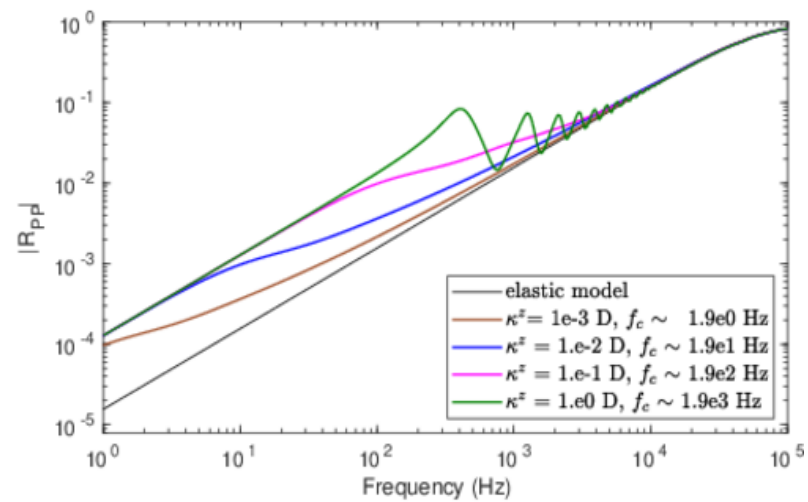

Figure 2. Absolute value of normal-incidence P-wave reflection coefficient $\left|R_{P P}\right|$ as a function of frequency for different DZ permeabilities $\kappa^{z}$.

Figures $3 \mathrm{a}$ and $3 \mathrm{~b}$ show the real and imaginary parts of the normal fracture compliance, respectively, as a function of frequency for different values of the DZ permeability $\kappa^{z}$. We use equation (24) to calculate both the elastic normal fracture compliance $Z_{N}^{e}$ and the poroelastic normal fracture compliance $Z_{N}^{p}$, respectively. As expected, the elastic normal compliance $Z_{N}^{e}$ is constant for all frequencies and presents the lowest compliance value, thus, indicating the undrained limit. In contrast, the poroelastic normal compliance $Z_{N}^{p}$ becomes complex-valued and frequency-dependent as the FPD regime transitions from the relaxed to the unrelaxed states. We point out that experimental support for the frequency-dependence of normal fracture compliance in poroelastic media has been provided by the work of Nakagawa (2013). This study presents results of fracture stiffness (inverse of compliance) as a function of frequency for a fluid-saturated fracture, showing curves with similar trends as those in Figure 3a. Notice that at the highfrequency limit, the real part of all poroelastic normal compliances $\operatorname{Re}\left[Z_{N}^{p}\right]$ (Figure 3a) converges to the value of the elastic normal compliance $Z_{N}^{e}$. This is because, at this frequency limit, there is not enough time for FPD to take place and the fracture behaves as hydraulically isolated. Regarding the behavior of normal fracture compliance at the low-frequency limit, Figure 3a shows that, at sufficiently low frequencies, the values of $\operatorname{Re}\left[Z_{N}^{p}\right]$ are highest since the fracture experiences the maximum deformation while the maximum fluid exchange occurs between the DZ and the fracture. Nonetheless, there is an upper limit for $\operatorname{Re}\left[Z_{N}^{p}\right]$ regardless of the $\mathrm{DZ}$ permeability, which is constrained by the pore volume available in the DZ for FPD. In addition, using equation (25), we obtain the fracture normal compliance for the low-frequency limit, $Z_{N}^{o}=3.4 \times 10^{-12} \mathrm{~m} / \mathrm{Pa}$ and the high-frequency limit, $Z_{N}^{u}=3.6 \times 10^{-13} \mathrm{~m} / \mathrm{Pa}$, respectively, which corresponds 
to a ratio $Z_{N}^{o} / Z_{N}^{u}$ equal to 9.45 . To corroborate the accuracy of these results, we also estimate the average normal compliance for these frequency limits directly from the plots presented in Figure 3a. To this end, we use the results from the curves with $k^{z}$ equal to $10^{-2} \mathrm{D}$ and $10^{-1} \mathrm{D}$ at a frequency of $1 \mathrm{~Hz}$ for the low-frequency limit and at a frequency of $4.5 \times 10^{4} \mathrm{~Hz}$ for the high-frequency limit. We perform the analysis with those two curves since they present both of the regimes relaxed and unrelaxed for the frequencies chosen. Although their compliances should be the same at these limits, we can expect minor precision errors due to floating numbers used for the computations, thus we report the average of the compliances. We obtain $3.39 \times 10^{-12} \mathrm{~m} / \mathrm{Pa}$ and $3.65 \times 10^{-13} \mathrm{~m} / \mathrm{Pa}$ for the average compliances in the low- and high-frequency limits, respectively. Comparing these results with the ones obtained using equation (25), we find that the errors are of the order of $1 \%$ or less. Moreover, as remarked for Figure 2, the DZ permeability controls the transition frequency towards the undrained normal compliance. The estimated values for the respective transition frequencies $f_{c}$ are presented in Figure 2. At this transition frequency, the magnitude of the imaginary part of fracture normal compliance has a peak (Figure $3 \mathrm{~b}$ ), which indicates that maximum energy dissipation is taking place. This is the result of FPD occurring at a characteristic length $h^{z}$ that has a comparable size to that of the diffusion length $L_{d}^{z}$ (equation (22)). Overall, these results indicate that FPD effects increase the normal fracture compliance as fluid exchange occurs between the fracture and the DZ, which, in turn, increases the reflectivity of the poroelastic fracture-DZ system.

\subsection{Effect of thickness and porosity of the DZ}

The thickness and porosity of the DZ determine the pore volume available for fluid flow due to FPD into the DZ. Thus, in the following examples (Figures 4 and 5), we show that, as the thickness and porosity of the DZ increase, so do FPD effects and, therefore, the maximum normal fracture compliance and the reflectivity of the fracture-DZ system. For the examples, we use the physical properties of Table 1, unless stated otherwise. We remark that, for the example in which we analyze the effect of changes in DZ porosity on reflectivity and compliance (Figure 5), we have disregarded the impact of porosity variations on the bulk modulus of the DZ. Although an increase in porosity is expected to decrease the bulk modulus correspondingly (e.g., Pride, 2003), we have neglected this effect to isolate the impact of porosity variations on reflectivity due to FPD. We remind the reader that the same consideration also applies to the elastic background since both DZ and background are assumed to have the same rock physical properties. 


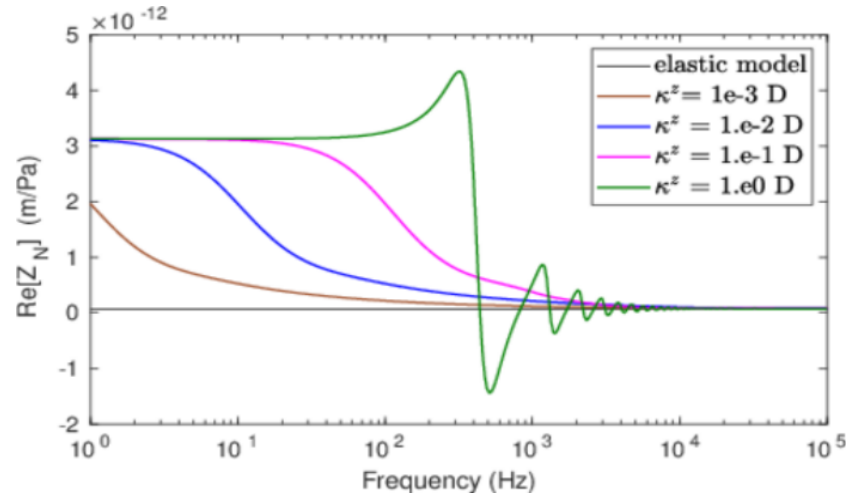

(a)

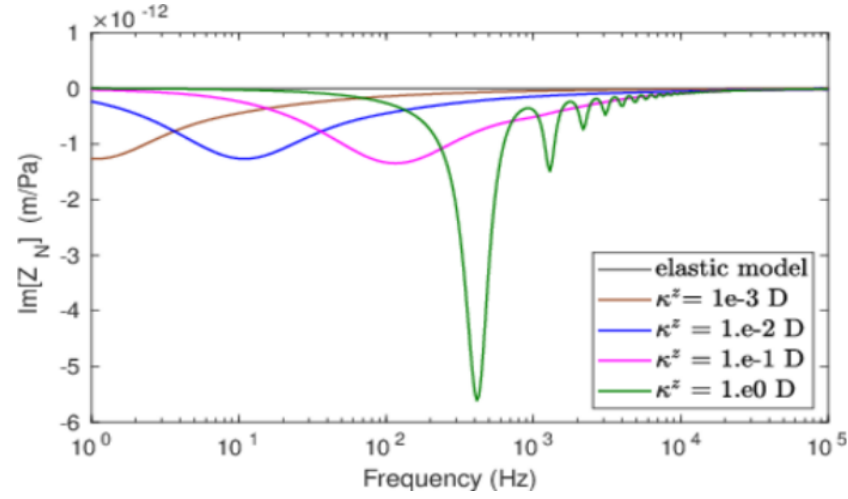

(b)

Figure 3. (a) Real and (b) imaginary parts of normal fracture compliance $Z_{N}$ as functions of frequency for different DZ permeabilities $\kappa^{z}$. 
Evidence from field data suggests that the thickness of DZ measured from the fault core can vary from centimeters to kilometers and is likely to scale with fault displacement (Mitchell \& Faulkner, 2009; Faulkner et al., 2011). In contrast, field measurements and estimations of the thickness of DZ surrounding magma driven veins are of the order of centimeters to meters (Engvik et al., 2005). For this example (Figure 4), we consider the effect on reflectivity and normal fracture compliance of variations of the DZ thickness of more than one order-of-magnitude, from $0.05 \mathrm{~m}$ to close to one meter $0.8 \mathrm{~m}$. These thicknesses would correspond to fault displacements of less than some tens of a meter (Mitchell \& Faulkner, 2009; Faulkner et al., 2011) and to fault lengths of less than one kilometer (Cowie \& Scholz, 1992). In particular, Figure 4a shows $\left|R_{P P}\right|$ as a function of frequency for a DZ permeability $\kappa^{z}$ of $0.1 \mathrm{D}$ and varying values of $\mathrm{DZ}$ thickness $h^{z}$. Figure $4 \mathrm{~b}$ shows the real part of $Z_{N}$ for the same DZ parameters. We notice that the maximum values of both $\left|R_{P P}\right|$ and $R e\left[Z_{N}\right]$ increase with increasing thickness $h^{z}$. This occurs because a wider DZ thickness provides more pore volume for FPD to prevail in its relaxed regime, and, as a consequence, more fluid is allowed to exit the fracture, thus, increasing its normal compliance and reflectivity. On the other hand, an increase in DZ thickness shifts the characteristic transition frequency $f_{c}$ towards lower values. This is expected since $f_{c}$ is inversely proportional to the square of thickness $h^{z}$ as shown by equation (23). We additionally remark that the compliance ratios $Z_{N}^{o} / Z_{N}^{u}$ are 32.51 and 3.1 for DZ thicknesses $h^{z}$ of $0.8 \mathrm{~m}$ and $0.05 \mathrm{~m}$, respectively. These results correspond to higher and lower values compared to the reference case (9.45), for which the thickness is $0.2 \mathrm{~m}$ (Table 1 and Figure 3a).

In Figure 5, we present results considering DZ and background porosities of 0.03 and 0.07 , respectively, to investigate the corresponding effects on reflectivity and normal fracture compliance. Specifically, Figure 5a shows $\left|R_{P P}\right|$ as a function of frequency for a DZ permeability $\kappa^{z}$ of $0.1 \mathrm{D}$ and varying values of $\mathrm{DZ}$ porosity $\phi^{z}$. Solid lines denote elastic-poroelastic models while dashed lines denote the corresponding elastic models. Figure $5 \mathrm{~b}$ shows the real part of $Z_{N}$ for the same DZ parameters. First, notice that results in Figure 5a indicate that the variations in porosity do not affect greatly the reflectivity of the respective elastic models. These results reveal the minor impact of porosity changes on the impedance $\rho_{b} V_{P}$ of the background and DZ. Indeed, the decrease in impedance is $\sim 1 \%$ for a porosity increase to 0.03 and of $\sim 2.4 \%$ for a porosity increase to 0.07 . The corresponding decrease in bulk density is $\sim 1 \%$ and $\sim 3.5 \%$, respectively. This is due to the very low value of Biot-Willis coefficient $\alpha \sim 0$, which prevents a change 
of porosity to affect significantly the undrained Lam modulus $\lambda$ (equation (3)) and, therefore, the $\mathrm{P}$-wave velocity. The reason for having $\alpha \sim 0$ is because the background bulk modulus (33 GPa) has a very similar value to that of the grain bulk modulus (37 GPa) (equation (3)). We also remark that it would be expected that the increase of the background porosity is associated with a decrease of the mechanical moduli. However, to be able to analyze the influence on reflectivity of variations of porosity only, we disregard its influence on the mechanical moduli. Notice also the similar effect that the increase of DZ porosity $\phi^{z}$ has on the results compared to that of the increase of its thickness $h^{z}$ : the higher the DZ porosity $\phi^{z}$, the higher the maximum value of reflectivity (Figure 5a) and of normal fracture compliance (Figure 5b). The same trend is reflected in the $Z_{N}^{o} / Z_{N}^{u}$ ratio, which presents increasing values of 15.96 and 32.35 that correspond to increasing DZ porosity $\phi^{z}$ of 0.03 and 0.07 , respectively. As already outlined, this is the effect of the greater pore volume that a higher DZ porosity provides for FPD. The transition frequency also presents a similar behavior to the one observed with increasing DZ thickness $h^{z}$ : the higher the DZ porosity $\phi^{z}$, the lower the transition frequency $f_{c}$. Nonetheless, the relationship of the transition frequency $f_{c}$ with porosity is not as evident as with thickness (equation 23), but the porosity is embedded in the relationship $M / H$, which is part of the formula to calculate the diffusion coefficient $D$ in equation (21).

\subsection{Effect of DZ mechanical moduli}

In this section, we study the effect of decreasing the drained bulk and shear moduli $K_{m}$ and $\mu$ of the $\mathrm{DZ}$ on reflectivity and normal fracture compliance. The material properties for the reference elastic model and elastic-poroelastic model are taken from Table 1. For all models, the background has the same rock properties of the DZ. For this example (Figure 6), we consider the decrease of the reference $K_{m}^{z}$ (Table 1) to $19.8 \mathrm{GPa}$ and $6.6 \mathrm{GPa}$, corresponding to $60 \%$ and $20 \%$ of its original value, respectively, while keeping a fixed $K_{m}^{z} / \mu^{z}$ ratio of 1.14. This ratio corresponds to that of the reference mechanical moduli. Solid lines in Figure 6a show $\left|R_{P P}\right|$ as a function of frequency for a DZ permeability $\kappa^{z}$ of $0.1 \mathrm{D}$ and varying values of DZ bulk modulus $K_{m}^{z}$. Dashed lines refer to the results of the corresponding elastic models. Figure $6 \mathrm{~b}$ shows the real part of $Z_{N}$ for the same DZ parameters. Note that the reflectivity of the elastic models decreases with decreasing bulk modulus of the background and DZ (Figure 6a). This is the result of the lower impedance contrast between the background and the DZ-fracture system produced by the decreasing values of the background and DZ mechanical moduli. On the other hand, the maximum increase of reflectivity due to FPD does not present such 


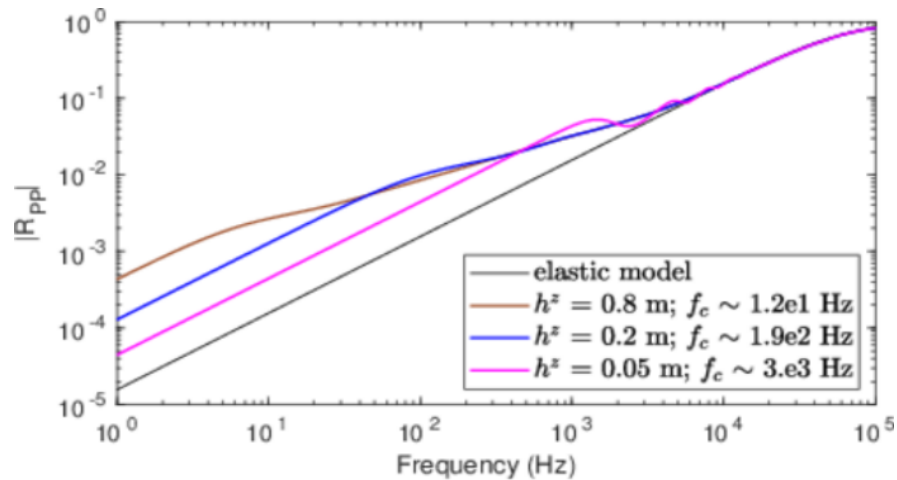

(a)

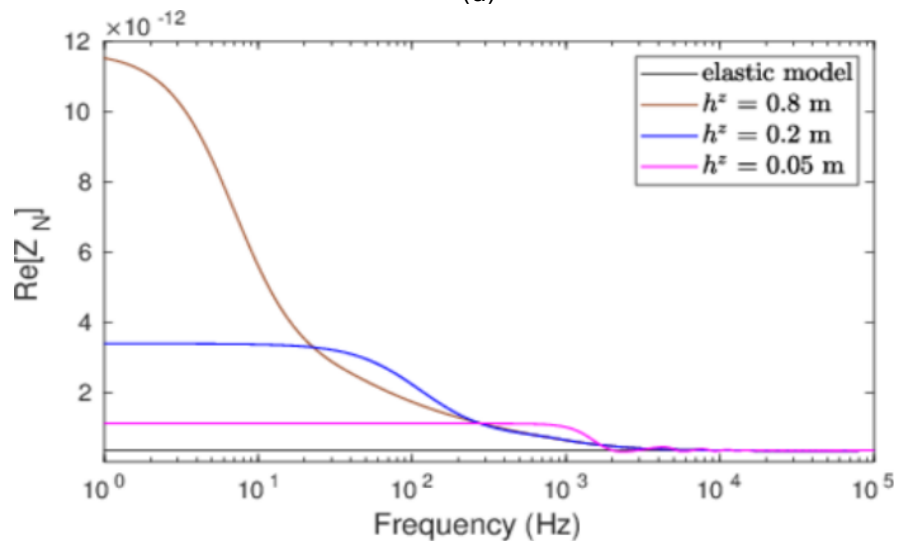

(b)

Figure 4. (a) Absolute value of normal-incidence P-wave reflection coefficient $\left|R_{P P}\right|$ and (b) real part of normal fracture compliance $Z_{N}$ as functions of frequency using a DZ permeability of $0.1 \mathrm{D}$ and different DZ thicknesses $h^{z}$. 


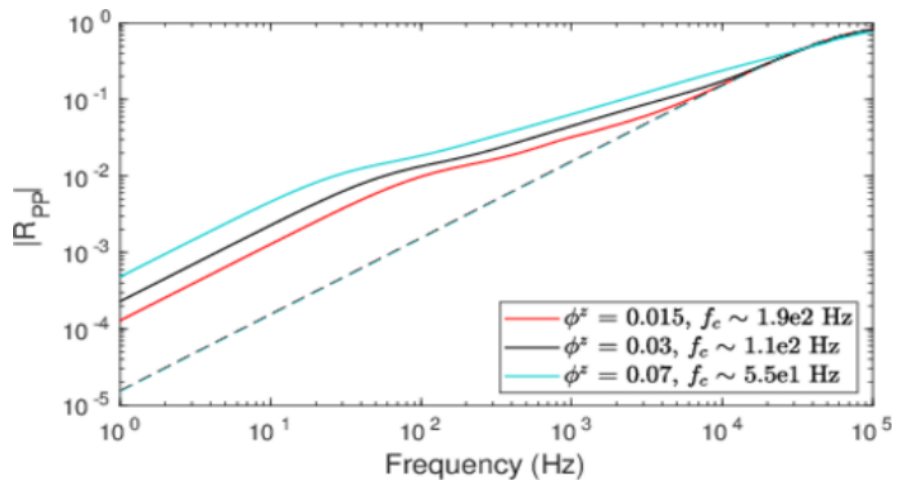

(a)

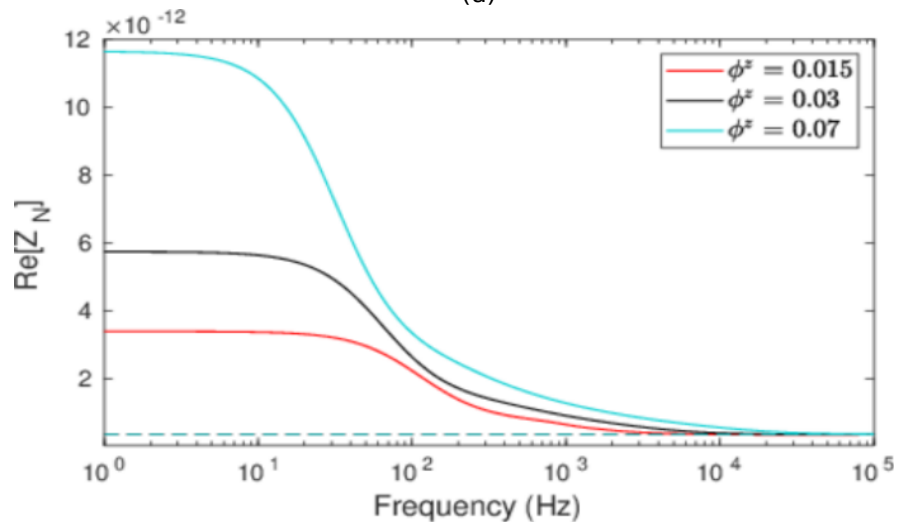

(b)

Figure 5. (a) Absolute value of normal-incidence P-wave reflection coefficient $\left|R_{P P}\right|$ and (b) real part of normal fracture compliance $Z_{N}$ as functions of frequency. Solid curves correspond to the elastic-poroelastic models generated using a DZ permeability of $0.1 \mathrm{D}$ and various DZ porosities $\phi^{z}$. For these models, the background has the same rock physical properties as the DZ. Dashed lines denote the corresponding elastic models. 
a monotonic trend. For a $K_{m}^{z}$ of $19.8 \mathrm{GPa}$, there is not appreciable difference in the maximum increase of reflectivity when compared to the reference case $\left(K_{m}^{z}=33 \mathrm{GPa}\right)$. On the contrary, for a $K_{m}^{z}$ of $6.6 \mathrm{GPa}$, it is evident that the maximum increase of reflectivity is much lower than for the other two cases. Regarding the impact on normal fracture compliance, Figure $6 \mathrm{~b}$ shows that decreasing the mechanical moduli results in a higher maximum increase of normal fracture compliance, which is an opposed effect to that on the maximum increase of reflectivity (Figure 6a). That is, that the decrease of the mechanical moduli, in general, decreases the maximum reflectivity of the DZ-fracture system. These opposed results occur because the decrease of the mechanical moduli has opposite effects on the induced FPD between the poroelastic fracture and associated DZ compared compared to its effect on the acoustic impedance contrast between the background and the DZ-fracture poroelastic system. The aforementioned impedance contrast decreases with decreasing $K_{m}^{z}$, producing a decrease in the maximum reflectivity of the elastic-poroelastic system. In contrast, Figure $6 \mathrm{~b}$ indicates that the decrease of mechanical moduli promotes FPD, which, in turn, has a positive impact on the maximum increase of normal fracture compliance. The latter can be explained by the opposed effects between the terms in the numerator and denominator involved in the calculation of the low-frequency limit of normal fracture compliance $Z_{N}^{o}$ (equation (25)). In the numerator, we have that as the DZ bulk modulus $K_{m}^{z}$ decreases, the DZ Skempton's coefficient $B^{z}$ increases, leading to lower values of $B^{c}-B^{z}$. This, in turn, decreases $Z_{N}^{o}$. However, in the denominator, we have that the term $M^{z}\left(1-\alpha^{z} B^{z}\right)$ decreases with lower values of $K_{m}^{z}$, which promotes a increase of $Z_{N}^{o}$. For the values of $K_{m}^{z}$ used in this example, in combination with the particular rock and fluid properties of Table 1, we find that the denominator has a stronger influence on $Z_{N}^{o}$ and it leads to a increase of the the maximum fracture normal compliance with decreasing $K_{m}^{z}$.

\subsection{Effect of fracture mechanical moduli}

Figures 7 and 8 show the effect of fracture thickness on reflectivity and normal compliance. However, this is equivalent to studying the effect of fracture moduli since both the thickness of the fracture and its mechanical moduli are related by equation (26). Specifically, Figures 7 and 8 show the effect of two fracture thicknesses, $5 \times 10^{-3} \mathrm{~m}$ and $2 \times 10^{-4}$ $\mathrm{m}$, respectively, on reflectivity and normal fracture compliance. To find the corresponding bulk and shear moduli, we use equation (26), keeping $Z_{T}^{d}$ and $Z_{N}^{d}$ constant and equal to $5 \times 10^{-10} \mathrm{~m} / \mathrm{Pa}$ and $1.5 \times 10^{-10} \mathrm{~m} / \mathrm{Pa}$, respectively. For the fracture with a thickness of $5 \times 10^{-3} \mathrm{~m}$, the corresponding values for $K_{m}$ and $\mu$ are $0.02 \mathrm{GPa}$ and $0.01 \mathrm{GPa}$. 


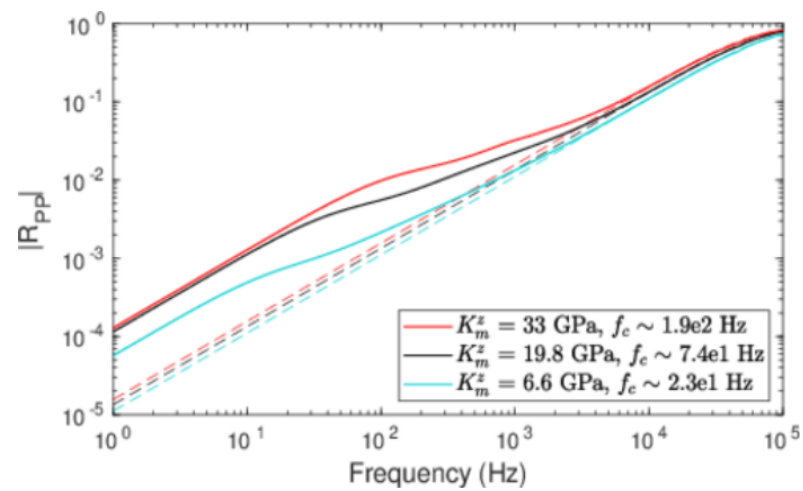

(a)

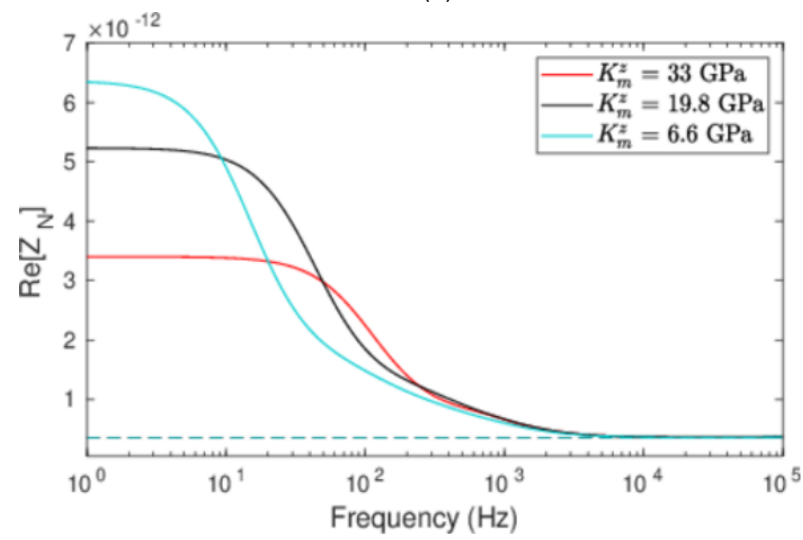

(b)

Figure 6. (a) Absolute value of normal-incidence P-wave reflection coefficient $\left|R_{P P}\right|$ and (b) real part of normal fracture compliance $Z_{N}$ as functions of frequency. Solid curves correspond to elastic-poroelastic models generated using a DZ permeability of $0.1 \mathrm{D}$ and different $\mathrm{DZ} K_{m}^{z}$ moduli with $K_{m}^{z} / \mu^{z}=1.14$. For these models the background has the same rock properties as the DZ. Dashed lines denote the corresponding elastic models. 


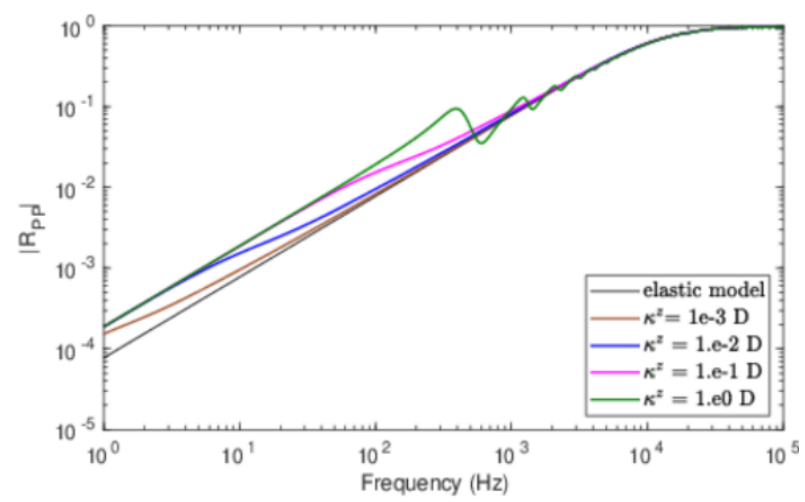

(a)

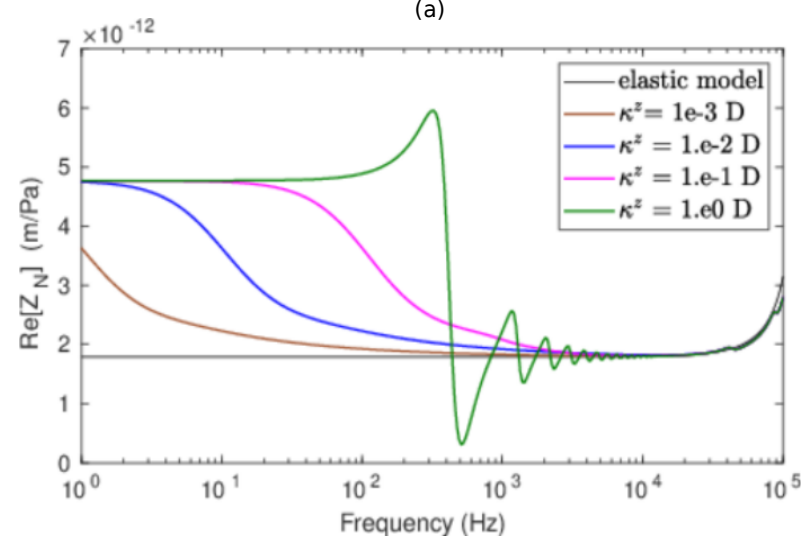

(b)

Figure 7. (a) Absolute value of normal-incidence P-wave reflection coefficient $\left|R_{P P}\right|$ and (b) real part of normal fracture compliance $Z_{N}$ as functions of frequency for different DZ permeabilities $\kappa^{z}$ for a fracture with a thickness of $5 \times 10^{-3} \mathrm{~m}$.

Similarly, for the fracture with a thickness of $2 \times 10^{-4} \mathrm{~m}$, the corresponding values for $K_{m}$ and $\mu$ are $8 \times 10^{-4} \mathrm{GPa}$ and $4 \times 10^{-4} \mathrm{GPa}$. When comparing the corresponding reflectivities $\left|R_{P P}\right|$ in Figures 7a and 8a, we find that it is higher for the thicker fracture. However, the maximum increase of reflectivity due to FPD effects is higher for the thinner fracture. This increase for the thinner fracture is more than one order-of magnitude (Figure 8a) compared to only a tenth of that increase for the thicker fracture (Figure 7a). A similar trend is also evident from the corresponding normal fracture compliance plots in Figures $7 \mathrm{~b}$ and $8 \mathrm{~b}$, with a larger increase of maximum normal compliance for the thinner and softer fracture. In fact, we find that the $Z_{N}^{o} / Z_{N}^{u}$ ratios are 2.67 and 43.35 for the thicker and thinner fractures, respectively. We also remark that the transition frequencies are the same as the ones shown in Figure 2 since we have not modified the properties of the DZ. 


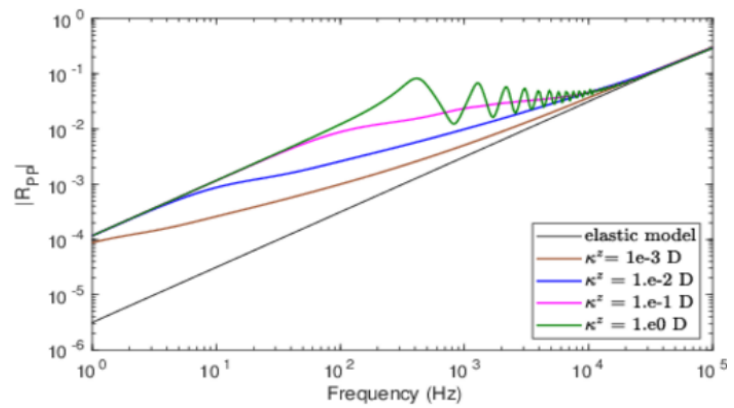

(a)

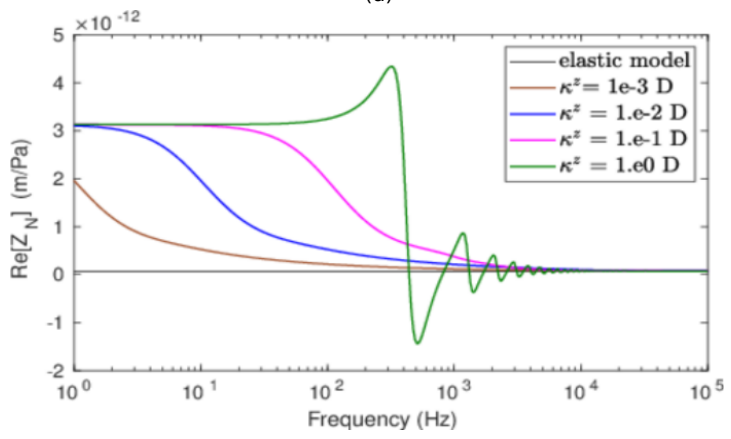

(b)

Figure 8. (a) Absolute value of normal-incidence P-wave reflection coefficient $\left|R_{P P}\right|$ and (b) real part of normal fracture compliance $Z_{N}$ as functions of frequency for different DZ permeabilities $\kappa^{z}$ for a fracture with a thickness of $2 \times 10^{-4} \mathrm{~m}$.

\subsection{Effect of a more compressible and less viscous pore fluid in the DZ and fracture}

Next, we study the effect of a more compressible and less viscous fluid, such as supercrital $\mathrm{CO}_{2}$ (Figure 9), filling the pores of the fracture and the associated DZ in both elastic-poroelastic and purely elastic models. We set the supercritcal $\mathrm{CO}_{2}$ properties $K_{f}$, $\rho_{f}$ and $\eta$ to $0.0229 \mathrm{GPa}, 693 \mathrm{Kg} / \mathrm{m}^{3}$ and $1.56 \times 10^{-5} \mathrm{~Pa} . \mathrm{s}$, respectively. These values are taken from Rubino and Velis (2011). Figure 9a shows that the elastic reflectivity obtained in such scenario is close to two orders-of-magnitude higher than the one obtained using water as the saturating fluid (Table 1 and Figure 2). However, the maximum increase of reflectivity due to FPD is less than half of that obtained with water as saturating fluid (Figure 2). A similar trend is observed for the normal fracture compliance, with higher values for the elastic normal compliance when using supercritical $\mathrm{CO}_{2}$ as the saturating fluid, of order of $1 \times 10^{-11} \mathrm{~m} / \mathrm{Pa}$, (Figure $9 \mathrm{~b}$ ) than for the case of water as the saturating fluid, order of $1 \times 10^{-12} \mathrm{~m} / \mathrm{Pa}$ (Figure 3a). Nonetheless, the maximum increase in compliance due to FPD effects is less for the case of $\mathrm{CO}_{2}$ as saturating fluid, 
as indicated by its lower $Z_{N}^{o} / Z_{N}^{u}$ ratio of 3.51 compared to the case of water as saturating fluid, with a higher $Z_{N}^{o} / Z_{N}^{u}$ ratio of 9.45 . Thus, even though the fracture-DZ poroelastic system saturated with $\mathrm{CO}_{2}$ is more seismically visible than its water-saturated counterpart, FPD effects are not as important. This lower increase in normal fracture compliance and reflectivity happens because $\mathrm{CO}_{2}$ has a much higher compressibility, of around two orders-of-magnitude, as compared to water. This prevents a significant increase of fluid pressure inside the fracture from taking place, even though the fracture is being heavily deformed. Therefore, the fluid pressure gradient between the fracture and the DZ is smaller, and so are the FPD effects. Another effect of considering supercritical $\mathrm{CO}_{2}$ as the pore fluid is the decrease of the transition frequency $f_{c}$ for a given DZ permeability, which is around $10 \%$ with respect to the water-saturated case. This is the result of the higher impact of the reduction of fluid compressibility compared to the impact of the decrease of its viscosity (equations (21) and (23)). We also observe the earlier onset of reverberations in Figure 9 than for the reference case (Figures 2 and $3 \mathrm{a}$ ) for the curves corresponding to $\mathrm{DZ}$ permeabilities of $1 \mathrm{D}$ and $1 \times 10^{-1} \mathrm{D}$, respectively. This is consequence of much lower values of Biot's frequency for the $\mathrm{DZ}$ of $\sim 1.8 \times 10^{1} \mathrm{~Hz}$ and $\sim 1.8 \times 10^{2}$ $\mathrm{Hz}$ for the respective DZ permeabilites, caused by the lower fluid viscosity.

\subsection{Sensitivity analysis of the maximum increase of normal fracture com- pliance}

We have shown in the previous examples the effect of discrete variations of rock and fluid properties of the DZ and fracture on the maximum increase of normal fracture compliance due to FPD. In this section, we investigate in more detail the sensitivity of the maximum increase of normal fracture compliance to the changes of rock and fluid properties. These properties are changed one at a time while keeping the other ones constant and equal to the values shown in Table 1.

We let the $Z_{N}^{o} / Z_{N}^{u}$ ratio be a measure of the maximum increase of normal fracture compliance due to FPD. According to equation (25), $Z_{N}^{o}$ is the low-frequency limit of normal compliance of the fracture. This means that it is the maximum value that it can take because at this frequency limit FPD is on its relaxed regime, causing the largest possible volume of fluid to exit the fracture. This, in turn, decreases to a minimum the fluid stiffening effect in the fracture. In contrast, $Z_{N}^{u}$ is the high-frequency limit of the normal compliance of the fracture, indicating that this is the lowest value that it can take because, at this frequency limit, the unrelaxed FPD regime prevails, which implies that 


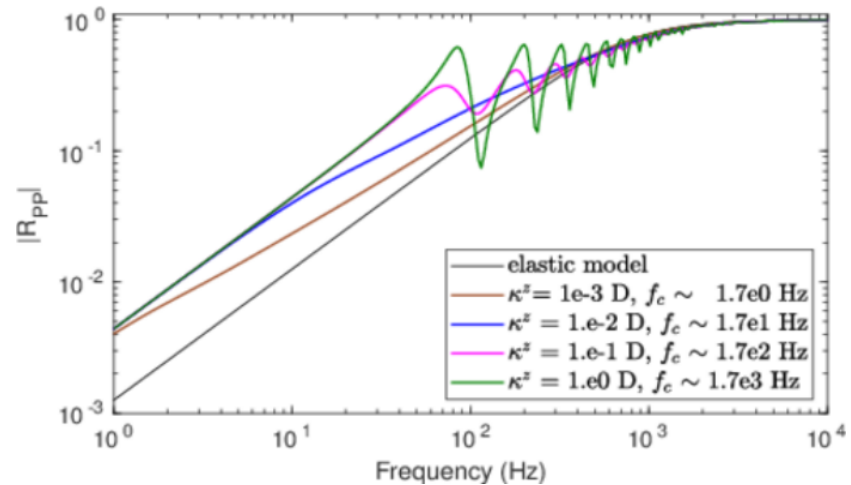

(a)

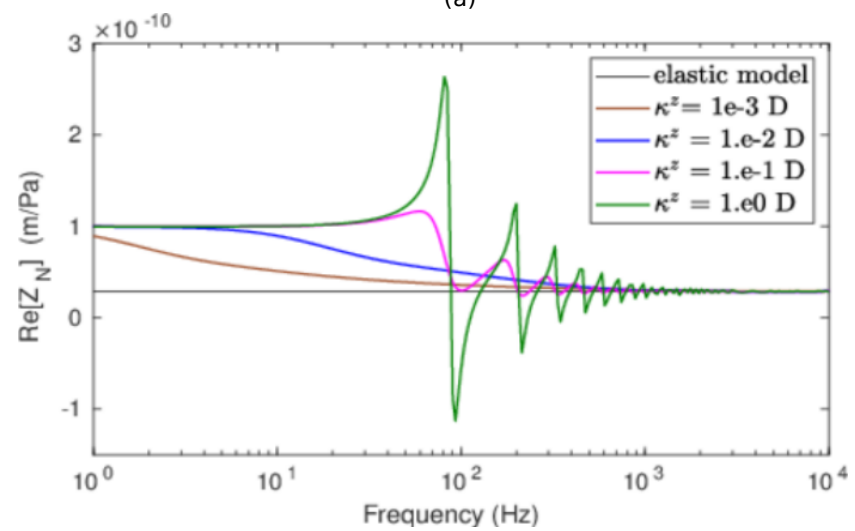

(b)

Figure 9. (a) Absolute value of normal-incidence P-wave reflection coefficient $\left|R_{P P}\right|$ and (b) real part of normal fracture compliance $Z_{N}$ as functions of frequency for different DZ permeabilities $\kappa^{z}$ considering supercritical $\mathrm{CO}_{2}$ as the saturating pore fluid for the fracture and associated DZ. 
the fracture behaves as hydraulically isolated. At this stage, the normal compliance value is that of an elastic fracture. Therefore, the $Z_{N}^{o} / Z_{N}^{u}$ ratio provides a measure of the maximum increase of normal fracture compliance due to FPD with respect to its elastic limit. To show how this maximum increase is controlled by the rock and fluid properties, we plot the $Z_{N}^{o} / Z_{N}^{u}$ ratio as a function of dimensionless properties $X$ (Figure 10), where $X$ indicates the factor by which a reference property value has increased. Table 2 lists the different dimensionless properties that $X$ represent as well as the corresponding reference value. We remark that the response of $Z_{N}^{o} / Z_{N}^{u}$ to the variations $\bar{K}_{m}^{c}$ and $\bar{K}_{m}^{z}$ also includes the effect of the respective shear moduli changes. Nonetheless, we only show the values that the dimensionless bulk moduli take. For the case of the fracture, we find both the bulk and shear modulus by means of equation (26). To this end, we vary the thickness of the fracture from $10^{-4} \mathrm{~m}$ to $10^{-2} \mathrm{~m}$, while keeping constant the tangential and drained normal compliance to $5 \times 10^{-10} \mathrm{~m} / \mathrm{Pa}$ and $1.5 \times 10^{-10} \mathrm{~m} / \mathrm{Pa}$, respectively. Then, the reference modulus $\tilde{K}_{m}^{c}$ corresponds to that found with a fracture thickness of $10^{-4} \mathrm{~m}$. For the case of the DZ, we simply assume that the bulk modulus is 1.14 times the value of the shear modulus. This ratio is the same as the one corresponding to the DZ moduli in Table 1.

Figure 10 shows that the increase of most of the dimensionless rock and fluid properties produces either a monotonic increase or decrease of the $Z_{N}^{o} / Z_{N}^{u}$ ratio. Properties producing an increase of $Z_{N}^{o} / Z_{N}^{u}$ as they increment are $\bar{\phi}^{z}, \bar{h}^{z}$ and $\bar{K}_{f}$. As already investigated in the previous examples, the increase of $\bar{\phi}^{z}$ and $\bar{h}^{z}$ has a positive impact on the maximum increase of normal fracture compliance because they provide a greater pore volume for FPD. On the other hand, an increasingly stiffer fluid $\bar{K}_{f}$, creates the necessary pressure gradient for FPD. In contrast, the increment of $\bar{K}_{m}^{c}$ produces a continuous decrease of the $Z_{N}^{o} / Z_{N}^{u}$ ratio because the fracture becomes increasingly stiffer. However, $\bar{K}_{m}^{z}$ is the only property, among the ones studied, that does not produce a monotonic response of the $Z_{N}^{o} / Z_{N}^{u}$ ratio. We observe that, for sufficiently low values of $\bar{K}_{m}^{z}$, the increase of this property causes a continuous rise of $Z_{N}^{o} / Z_{N}^{u}$ until a maximum is reached. Then, a further increase of $\bar{K}_{m}^{z}$ produces a continuous decline of the $Z_{N}^{o} / Z_{N}^{u}$ ratio. The non-monotonic behavior of $Z_{N}^{o} / Z_{N}^{u}$ occurs as a consequence of the opposing effects that $B^{z}$ and $M^{Z}$ have on the DZ fluid pressure $p_{f}^{z}=-B^{z} H^{z} \nabla \cdot \boldsymbol{u}^{s}-M^{z} \nabla \cdot \boldsymbol{w}$. That is, increasing values of $\bar{K}_{m}^{z}$ decreases $B^{z}$ and $B^{z} H^{z}$, and this, in turn, induces lower magnitudes of $p_{f}^{z}$, which will tend to promote higher pressure gradients for FPD, and as a consequence, higher values of $Z_{N}^{o}$. On the contrary, increasing values of $\bar{K}_{m}^{z}$ increases 
$M^{z}$. This, in turn, induces higher magnitudes of $p_{f}^{z}$, which will tend to promote lower pressure gradients for FPD and, as a consequence, a reduction of $Z_{N}^{o}$. In section 3.3, we have analyzed the effects of DZ moduli on the maximum increase of fracture normal compliance. In that analysis we have found that for values of $K_{m}^{z} \geq 6.6 \mathrm{GPa}\left(\bar{K}_{m}^{z} \geq 33\right)$, the maximum increase of normal fracture compliance decreases with the increase of $K_{m}^{z}$. This means that for the $K_{m}^{z}$ values used in that section, the response of the $Z_{N}^{o} / Z_{N}^{u}$ ratio is in the decreasing part of the curve. Notice that, for this sensitivity analysis, we do not consider neither the permeability of the DZ nor the viscosity of the saturating fluid, because, according to equation (25)), none of these properties has any effect on the maximum increase on normal compliance. Nonetheless, these parameters control the transition frequency between FPD regimes (equations (21) and (23)).

For completeness, Table 3 shows the $Z_{N}^{o} / Z_{N}^{u}$ ratios for the rock and fluid properties analyzed in the previous sections. Here, the column Marker refers to the marker used in Figure 10 to plot the respective data entry. These results can be compared against the $Z_{N}^{o} / Z_{N}^{u}$ ratio of 9.45 obtained for the reference elastic-poroelastic model using the properties of Table 1. The dimensionless variables of interest for the rock and fluid properties of Table 1 are $\bar{h}^{z}=20, \bar{K}_{m}^{z}=165, \bar{K}_{m}^{c}=10$ and $\bar{K}_{f}^{z}=225$.

Table 2. Definition of the dimensionless properties and the corresponding reference values used for Figure 10 .

\begin{tabular}{|l|l|}
\hline Dimensionless property $X$ & Reference value \\
\hline Increment of fracture bulk modulus $\bar{K}_{m}^{c}$ & $\tilde{K}_{m}^{c}=4 \times 10^{-4} \mathrm{GPa}$ \\
Increment of DZ bulk modulus $\bar{K}_{m}^{z}$ & $\tilde{K}_{m}^{z}=0.2 \mathrm{GPa}$ \\
Increment of DZ porosity $\bar{\phi}^{z}$ & $\tilde{\phi}^{z}=0.01$ \\
Increment of DZ thickness $\bar{h}^{z}$ & $\tilde{h}^{z}=0.01 \mathrm{~m}$ \\
Increment of fluid bulk modulus $\bar{K}_{f}$ & $\tilde{K}_{f}=0.01 \mathrm{GPa}$ \\
\hline
\end{tabular}

\section{Discussion}

In this work, we have shown that the presence of a DZ in low-permeability formations has the potential to increase the compliance and reflectivity of a fracture due to FPD in the seismic exploration frequency range. Specifically, our study indicates that the rock and fluid physical properties of the DZ and fracture have a direct control on 


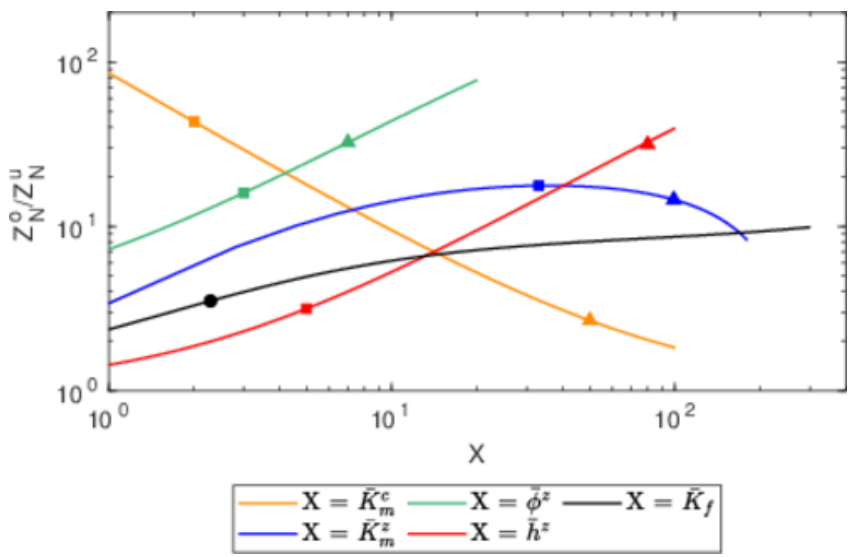

Figure 10. $Z_{N}^{o} / Z_{N}^{u}$ ratio as a function of dimensionless rock and fluid properties $X$ obtained with normalization with corresponding reference values. Property $X$ represents the factor by which a reference value has increased. The corresponding dimensionless properties are: the increment of the fracture bulk modulus $\bar{K}_{m}^{c}$, the increment of the DZ bulk modulus $\bar{K}_{m}^{z}$, the increment of DZ thickness $\bar{h}^{z}$ and the increment of the fluid bulk modulus $\bar{K}_{f}$. The corresponding reference values are: $\tilde{K}_{m}^{c}=4 \times 10^{-4} \mathrm{GPa}, \tilde{k}_{m}^{z}=0.2 \mathrm{GPa}$, the thickness of $\mathrm{DZ} h^{z}=0.01 \mathrm{~m}$ and $K_{f}=0.01 \mathrm{GPa}$. Markers denote data points as detailed in Table 3.

the fluid exchange between these two regions due to FPD and, therefore, they determine the maximum increase of normal fracture compliance from its elastic limit. However, the variation of rock properties may not produce the same trend on reflectivity as they do on the normal fracture compliance because these property changes may cause opposing results on FPD between the fracture and DZ and on the impedence contrast between the background and the poroelastic fracture-DZ system. This response is observed, for instance, when decreasing the DZ and background mechanical moduli (Figure 8). For the elastic-poroelastic models tested in that example, the maximum fracture normal compliance decreases but the maximum reflectivity in general increases with increasing values of $K_{m}^{z}$. In fact, it is possible to show that the maximum acoustic impedance contrast between the background and the poroelastic fracture-DZ system increases with $K_{m}^{z}$. To this end, the low-frequency limit P-wave velocity of the poroelastic DZ-fracture system can be calculated as suggested by Brajanovski et al. (2005). We remind the reader that for these examples the mechanical moduli of the background is the same as those for the DZ. A corollary of these observations is that a 1D model considering a slip interface characterized by a poroelastic normal compliance, as the ones calculated in the 
Table 3. $Z_{N}^{o} / Z_{N}^{u}$ ratio for the different rock and fluid properties studied in the previous examples. See Table 2 for a description of the dimensionless properties and Figure 10 for the plots of the data points.

\begin{tabular}{|l|c|c|c|}
\hline Property (dimensionless) & Property value (dimensionless) & $Z_{N}^{o} / Z_{N}^{u}$ & Marker \\
\hline$h^{z}\left(\bar{h}^{z}\right)$ & $0.05 \mathrm{~m}(5)$ & 3.15 & $\mathbf{0}$ \\
\hline$\phi^{z}\left(\bar{\phi}^{z}\right)$ & $0.8 \mathrm{~m}(80)$ & 31.51 & $\Delta$ \\
\hline$K_{m}^{z}\left(\bar{K}_{m}^{z}\right)$ & $0.03(3)$ & 15.96 & \\
\hline$K_{m}^{c}\left(\bar{K}_{m}^{c}\right)$ & $0.07(7)$ & 32.35 & $\Delta$ \\
\hline$K_{f}\left(\bar{K}_{f}\right)$ & $19.8 \mathrm{GPa}(99)$ & 14.53 & $\mathbf{A}$ \\
\hline
\end{tabular}

examples presented in this work, does not, in principle, represent the seismic response of the poroelastic fracture-DZ system, since the acoustic impedance of this entire system is not accounted for. Nonetheless, we expect to explore models that are seismically equivalent to the aforementioned poroelastic system in future works.

We have considered that the $\mathrm{DZ}$ and the adjacent impermeable background have the same rock properties except for the permeability, since we have aimed to highlight the effects of FPD on reflectivity. Thus, we have not analyzed the effect on reflectivity of any decrease in mechanical moduli or increase in porosity in the DZ with respect to the background, although these effects are expected due to the presence of micro- and macro-fractures in the DZ. In Figure 11, we present such an analysis. Here, solid lines correspond to elastic-poroelastic models for a DZ permeability of $0.1 \mathrm{D}$ and dashed curves of the same color denote the corresponding elastic model. Unless stated otherwise, all other DZ properties are the same as in Table 1. Figure 11a shows the effect of the decrease of DZ bulk and shear moduli while the corresponding background moduli are kept constant. The red solid curve corresponds to the elastic-poroelastic model, for which the background and DZ have the same rock and fluid properties. The red dashed curve shows the reflectivity for the corresponding elastic model. We observe that, compared to this 
elastic model, the other two present higher reflectivities. The reason for this increase in reflectivity is the presence of a softer region comprised by the DZ and fracture that produces a higher impedance contrast with regard to the background: the elastic reflectivity increases as the DZ becomes softer. In contrast, the maximum increase of reflectivity due to FPD from its corresponding elastic reference decreases as the DZ becomes softer. This is the consequence of the decreasing mechanical contrast between the DZ and the fracture. Nonetheless, it is likely that the DZ becomes not only softer but also more porous. Figure 11b presents reflectivities of models considering increasing porosities of a DZ that is softer than the background. As expected, the increase of the pore volume promotes FPD, increasing the maximum reflectivity from its elastic reference, thus counteracting the effect of the decrease of DZ bulk and shear moduli. We assume that changes in porosity do not have any further effect on the bulk modulus of the DZ, although it is expected that an increase in porosity would decrease the bulk modulus. On the one hand, this would result in a higher impedance contrast with the stiffer background rock, on the other hand, however, the softening of the DZ bulk modulus would decrease the FPD effects between the fracture and DZ (Figure 10).

We have shown that the FPD effects between an isolated fracture and its surroundings (DZ) even in largely impermeable rocks are evidenced by the fact that the normal fracture compliance becomes complex-valued, presenting the largest magnitude of its imaginary part when the energy dissipation is the greatest. This result provides a possible explanation for the existence of an imaginary part in seismic measurements even if the background is largely impermeable (Barbosa et al., 2019). Furthermore, our results regarding the enhanced reflectivity in the seismic frequency band further imply that FPD between the fracture and its associated DZ could be an important factor, for which reflectivity from fractures can be distinguished using seismic exploration techniques even in largely impermeable environments (e.g., Kim et al., 1994; Schmelzbach et al., 2007).

Future research should consider more realistic configurations of the DZ. For instance, these models should include the effect of discrete fractures in the DZ. However, to be able to calculate the reflectivity with a semi-analytical approach of a poroelastic system comprised by an isolated fractured and such a complex DZ representation, it would be necessary to upscale this system using techniques such as the one proposed by Rubino et al. (2016). The isolated fracture and associated complex DZ could then be represented by an equivalent anisotropic viscoelastic medium. Another approach would be to consider the fracture-complex DZ poroelastic system as an equivalent viscoelastic slip in- 


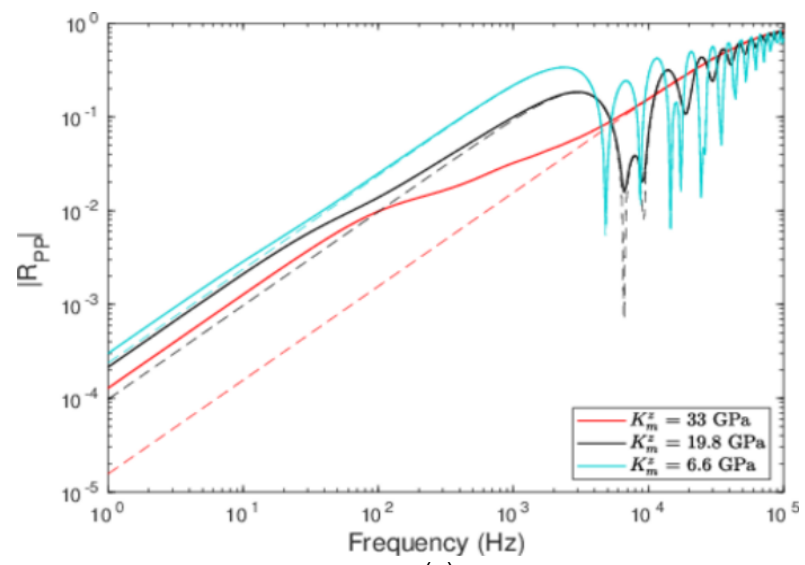

(a)

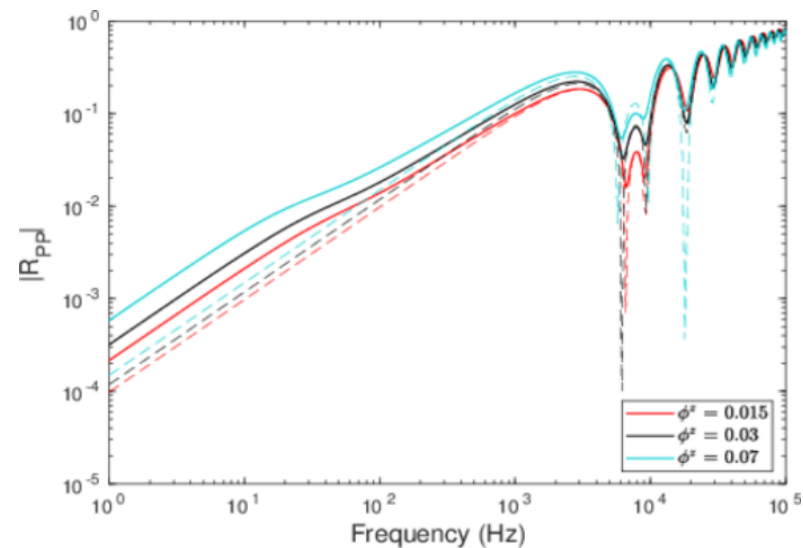

(b)

Figure 11. Absolute value of normal-incidence P-wave reflection coefficient $\left|R_{P P}\right|$ as a function of frequency. Solid lines correspond to elastic-poroelastic models for a DZ permeability of 0.1 D. Dashed lines of the same color denote the corresponding elastic models. (a) Curves for varying values of DZ bulk moduli $k_{m}^{z}$ with $k_{m}^{z} / \mu^{z}=1.14$. The background bulk modulus is kept constant to $33 \mathrm{GPa}$. (b) Curves for varying values of DZ porosity $\phi^{z}$. DZ bulk modulus $k_{m}^{z}$ is $19.8 \mathrm{GPa}$. 
terface. Meaning that the entire poroelastic system is modeled as a displacement-jump interface characterized by complex-valued, frequency-dependent compliances. However, this representation would be valid only for poroelastic fracture-DZ systems with thicknesses much smaller than the prevailing seismic wavelengths.

\section{Conclusions}

We have considered a layered model to analyze the poroelastic effects associated with a DZ adjacent to a fracture in a low-permeability background rock. Our results show that FPD between a fracture and its adjacent DZ increases fracture normal compliance, as this process allows fluid pressure release from the fracture into the DZ. As a consequence, the reflectivity of the system also increases compared to an impermeable reference model. Our results also show that the maximum increase of normal compliance and reflectivity are most sensitve to the increase in DZ thickness and porosity as well as to to the decrease of fracture mechanical moduli. In contrast, the permeability of the DZ does not have any effect in the maximum increase of reflectivity but controls the transition frequency between FPD regimes and, therefore, constrains the visibility of the FPD effects on reflectivity: the greater the permeability of the DZ, the higher the transition frequency to the unreleaxed FPD regime, which allows for a wider range of frequencies for FPD to contribute in its relaxed regime. The thickness and porosity of the DZ affect both the maximum increase of reflectivity and the transition frequency. Greater thicknesses and porosities increase the reflectivity of the system but shift the transition frequency to lower values. The consequence of this latter is that the visibility of FPD effects on reflectivity is constrained to lower frequency bands. In this regard, an increase of the DZ thickness and porosity has an opposing effect to that of an increase of the DZ permeability. Regarding the effect of decreasing the mechanical moduli of the DZ, our results show that this decrease limits to lower values the maximum increase of reflectivity due to FPD. However, this effect is opposed by a likely increase of DZ porosity. Overall, this study shows that FPD effects promoted by the presence of a DZ in an otherwise largely impermeable background can notably enhance the reflectivity of a fracture in the seismic frequency band.

\section{Acknowledgments}

The data set used to plot the figures presented in this paper can be found at https:// doi.org/10.5281/zenodo.4085397. This data set has been generated by solving the 
system of equations comprised of equation (15) to equation (20). The reference input parameters are as detailed in Table 1 . This work is supported by grant number 200020178946 from the Swiss National Science Foundation and has been completed within the Swiss Competence Center on Energy Research Supply of Electricity, with the support of Innosuisse. J. G. R. gratefully acknowledges the financial support received from the Agencia Nacional de Promocin Cientfica y Tecnolgica of Argentina (PICT 2017-2976).

\section{References}

Bakulin, A., Grechka, V., \& Tsvankin, I. (2000). Estimation of fracture parameters from reflection seismic data - Part I: HTI model due to a single fracture set. Geophysics, 65(6), 1788-1802.

Barbosa, N. D., Caspari, E., Rubino, J. G., Greenwood, A., Baron, L., \& Holliger, K. (2019). Estimation of fracture compliance from attenuation and velocity analysis of full-waveform sonic log data. Journal of Geophysical Research: Solid Earth, 124(3), 2738-2761. doi: 10.1029/2018JB016507

Barbosa, N. D., Rubino, J. G., Caspari, E., \& Holliger, K. (2017). Extension of the classical linear slip model for fluid-saturated fractures: Accounting for fluid pressure diffusion effects. Journal of Geophysical Research: Solid Earth, 122(2), 1302-1323. doi: 10.1002/2016JB013636

Barbosa, N. D., Rubino, J. G., Caspari, E., Milani, M., \& Holliger, K. Fluid pressure diffusion effects on the seismic reflectivity of a single fracture. The Journal of the Acoustical Society of America, 140(4), 2554-2570. doi: $10.1121 / 1.4964339$

Biot, M. A. (1962). Mechanics of deformation and acoustic propagation in porous media. Journal of Applied Physics, 33(4), 1482-1498. doi: 10.1063/1.1728759

Brace, W. F. $\quad$ (1984). Permeability of crystalline rocks: New in situ measurements. Journal of Geophysical Research: Solid Earth, 89(B6), 4327-4330. doi: 10.1029/JB089iB06p04327

Braester, C. (1999). Radioactive waste repositories in fractured rocks formations: Hydrodynamic aspects. In P. Bejan Adrian and Vadász \& K. D. G (Eds.), Energy and the environment (pp. 229-238). Dordrecht: Springer Netherlands. doi: 10.1007/978-94-011-4593-0_20

Brajanovski, M., Gurevich, B., \& Schoenberg, M. (2005). $\quad$ A model for Pwave attenuation and dispersion in a porous medium permeated by aligned 
fractures. Geophysical Journal International, 163(1), 372-384.

doi: 10.1111/j.1365-246X.2005.02722.X

Brajanovski, M., Müller, T. M., \& Gurevich, B. (2006). Characteristic frequencies of seismic attenuation due to wave-induced fluid flow in fractured porous media. Geophysical Journal International, 166(2), 574-578. $\quad$ doi: 10.1111/j.1365-246X.2006.03068.X

Brown, R. J., Kavanagh, J., Sparks, R. S., Tait, M., \& Field, M. (2007). Mechanically disrupted and chemically weakened zones in segmented dike systems cause vent localization: Evidence from kimberlite volcanic systems. Geology, 35(9), 815-818. doi: 10.1130/G23670A.1

Chandler, R. N., \& Johnson, D. L. (1981). The equivalence of quasistatic flow in fluid-saturated porous media and Biot's slow wave in the limit of zero frequency. Journal of Applied Physics, 52(5), 3391-3395. doi: 10.1063/1.329164

Charlaix, E., Kushnick, A. P., \& Stokes, J. P. (1988). Experimental study of dynamic permeability in porous media. Phys. Rev. Lett., 61(14), 1595-1598. doi: 10.1103/PhysRevLett.61.1595

Cowie, P. A., \& Scholz, C. H. (1992). Displacement-length scaling relationship for faults: data synthesis and discussion. Journal of Structural Geology, 14(10), 1149-1156. doi: 10.1016/0191-8141(92)90066-6

Dahi Taleghani, A., Olson, J. E., \& Others. (2013). How natural fractures could affect hydraulic-fracture geometry. SPE journal, 19(01), 161-171.

Delaney, P. T., \& Pollard, D. D. (1981). Deformation of host rocks and flow of magma during growth of minette dikes and breccia-bearing intrusions near Ship Rock, New Mexico. U.S. Geological Survey Professional Papers, 1202. doi: $10.3133 / \mathrm{pp} 1202$

Deresiewicz, H., \& Skalak, R. (1963). On uniqueness in dynamic poroelasticity. Bulletin of the Seismological Society of America, 53(4), 783-788.

Engvik, A. K., Bertram, A., Kalthoff, J. F., Stöckhert, B., Austrheim, H., \& Elvevold, S. (2005). Magma-driven hydraulic fracturing and infiltration of fluids into the damaged host rock, an example from Dronning Maud Land, Antarctica. $\quad$ Journal of Structural Geology, 27(5), 839-854. $\quad$ doi: 10.1016/j.jsg.2005.01.009

Fang, X., Zheng, Y., \& Fehler, M. C. (2017). Fracture clustering effect on amplitude variation with offset and azimuth analyses. Geophysics, 82(1), N13-N25. doi: 10.1190/geo2016-0045.1 
Faulkner, D., Mitchell, T., Jensen, E., \& Cembrano, J. ～(2011). Scaling of fault damage zones with displacement and the implications for fault growth processes. Journal of Geophysical Research, 116(B5), B05403. doi: 10.1029/2010JB007788

Gale, J. F. W., \& Holder, J. (2010). Natural fractures in some US shales and their importance for gas production. In Geological society, london, petroleum geology conference series (Vol. 7, pp. 1131-1140).

Gassmann, F. (1951). Elastic waves through a packing of spheres. Geophysics, 16(4), 673-685. doi: 10.1190/1.1437718

Gu, B., Suárez-Rivera, R., Nihei, K. T., \& Myer, L. R. (1996). Incidence of plane waves upon a fracture. Journal of Geophysical Research: Solid Earth, 101 (B11), 25337-25346.

He, Y., Wang, S., Wu, X., \& Xi, B. (2020). Influence of frequencydependent anisotropy on seismic amplitudeversusoffset signatures for fractured poroelastic rocks. Geophysical Prospecting, 68(7), 2141-2163. doi: 10.1111/ $1365-2478.12981$

Hobday, C., \& Worthington, M. H. (2012). Field measurements of normal and shear fracture compliance. Geophysical Prospecting, 60(3), 488-499. doi: 10.1111/j $.1365-2478.2011 .01000 . x$

Jaeger, J. C., Cook, N. G. W., \& Zimmerman, R. (2009). Hydromechanical behavior of fractures. In Fundamentals of rock mechanics. John Wiley \& Sons.

Johnson, D. L., Koplik, J., \& Dashen, R. (1987). Theory of dynamic permeability and tortuosity in fluid-saturated porous media. Journal of Fluid Mechanics, 176(-1), 379. doi: 10.1017/S0022112087000727

Kim, J. S., Moon, G., Wooil M.and Lodha, Serzu, M., \& Soonawala, N. Imaging of reflection seismic energy for mapping shallow fracture zones in crystalline rocks. Geophysics, 59(5), 753-765. doi: 10.1190/1.1443633

Kim, J. S., Peacock, D. C., \& Sanderson, D. J. (2004). Fault damage zones. Journal of Structural Geology, 26(3), 503-517. doi: 10.1016/j.jsg.2003.08.002

Liu, E. (2005). Effects of fracture aperture and roughness on hydraulic and mechanical properties of rocks: implication of seismic characterization of fractured reservoirs. Journal of Geophysics and Engineering, 2(1), 38-47. doi: $10.1088 / 1742-2132 / 2 / 1 / 006$

Minato, S., \& Ghose, R. (2013). Inverse scattering solution for the spatially heterogeneous compliance of a single fracture. Geophysical Journal International, 
195(3), 1878-1891. doi: 10.1093/gji/ggt348

Minato, S., \& Ghose, R. (2016). Enhanced characterization of fracture compliance heterogeneity using multiple reflections and data-driven Green's function retrieval. Journal of Geophysical Research: Solid Earth, 121(4), 2813-2836. doi: 10.1002/2015JB012587

Mitchell, T., \& Faulkner, D. (2009). The nature and origin of off-fault damage surrounding strike-slip fault zones with a wide range of displacements: A field study from the atacama fault system, northern chile. Journal of Structural Geology, 31(8), 802-816. doi: 10.1016/j.jsg.2009.05.002

Mitchell, T., \& Faulkner, D. (2012). Towards quantifying the matrix permeability of fault damage zones in low porosity rocks. Earth and Planetary Science Letters, 339-340, 24-31. doi: 10.1016/J.EPSL.2012.05.014

Müller, T. M., Gurevich, B., \& Lebedev, M. (2010). Seismic wave attenuation and dispersion resulting from wave-induced flow in porous rocks a review.

Geophysics, 75(5), 75A147-75A164. doi: 10.1190/1.3463417

Müller, T. M., \& Rothert, E. (2006). Seismic attenuation due to wave-induced flow: Why Q in random structures scales differently. Geophysical Research Letters, 33(16), L16305. doi: 10.1029/2006GL026789

Nakagawa, S. (2013). Low-frequency $(<100 \mathrm{~Hz})$ dynamic fracture compliance measurement in the laboratory. In 47th Rock Mechanics / Geomechanics Symposium. San Fracisco: American Rock Mechanics Association.

Nakagawa, S., \& Schoenberg, M. A. (2007). Poroelastic modeling of seismic boundary conditions across a fracture. The Journal of the Acoustical Society of America, 122(2), 831-847. doi: 10.1121/1.2747206

Norris, A. N. (1993). Low-frequency dispersion and attenuation in partially saturated rocks. Journal of the Acoustical Society of America, 94(1), 359-370. doi: $10.1121 / 1.407101$

Ofterdinger, U., MacDonald, A. M., Comte, J.-C., \& Young, M. E. (2019). Groundwater in fractured bedrock environments: managing catchment and subsurface resources an introduction. Geological Society, London, Special Publications, 479(1), 1-9. doi: 10.1144/SP479-2018-170

Ogata, K., Senger, K., Braathen, A., Tveranger, J., \& Olaussen, S. (2014). The importance of natural fractures in a tight reservoir for potential $\mathrm{CO} 2$ storage: a case study of the upper Triassicmiddle Jurassic Kapp Toscana Group (Spitsbergen, Arctic Norway). In Advances in the study of fractured reservoirs. 
Geological Society of London. doi: 10.1144/SP374.9

Pride, S. R. (2003). Relationships between seismic and hydrological properties. In Y. Rubin \& S. Hubbard (Eds.), Hydrogeophysics (pp. 253-290). Dordrecht: Springer Netherlands. doi: 10.1007/1-4020-3102-5_9

Pyrak-Nolte, L. J., Myer, L. R., \& Cook, N. G. W. (1990). Transmission of seismic waves across single natural fractures. Journal of Geophysical Research, 95(B6), 8617. doi: 10.1029/JB095iB06p08617

Rubino, J. G., Caspari, E., Müller, T. M., Milani, M., Barbosa, N. D., \& Holliger, K. (2016). Numerical upscaling in 2-D heterogeneous poroelastic rocks: Anisotropic attenuation and dispersion of seismic waves. Journal of Geophysical Research: Solid Earth, 121(9), 6698-6721. doi: 10.1002/2016JB013165

Rubino, J. G., Castromán, G. A., Müller, T. M., Monachesi, L. B., Zyserman, F. I., \& Holliger, K. (2015). Including poroelastic effects in the linear slip theory. Geophysics, 80(2), A51-A56. doi: 10.1190/geo2014-0409.1

Rubino, J. G., Müller, T. M., Guarracino, L., Milani, M., \& Holliger, K. (2014). Seismoacoustic signatures of fracture connectivity. Journal of Geophysical Research: Solid Earth, $119(3), 2252-2271$. doi: 10.1002/2013JB010567

Rubino, J. G., \& Velis, D. R. (2011). S Seismic characterization of thin beds containing patchy carbon dioxide-brine distributions: A study based on numerical simulations. Geophysics, 76 (3), R57-R67. doi: 10.1190/1.3556120

Rüger, A. (1998). Variation of P-wave reflectivity with offset and azimuth in anisotropic media. Geophysics, 63(3), 935-947. doi: 10.1190/1.1444405

Savage, H. M., \& Brodsky, E. E. (2011). Collateral damage: Evolution with displacement of fracture distribution and secondary fault strands in fault damage zones. Journal of Geophysical Research: Solid Earth, 116(3). doi: 10.1029/2010JB007665

Schmelzbach, C., Horstmeyer, H., \& Juhlin, C. (2007). Shallow 3D seismic-reflection imaging of fracture zones in crystalline rock. Geophysics, 72(6), B149-B160. doi: $10.1190 / 1.2787336$

Schoenberg, M. (1980). Elastic wave behavior across linear slip interfaces. The Journal of the Acoustical Society of America, 68(5), 1516-1521.

Sheng, P., \& Zhou, M.-Y. (1988). Dynamic permeability in porous media. Phys. Rev. Lett., 61(14), 1591-1594. doi: 10.1103/PhysRevLett.61.1591

Smeulders, D. M. J., Eggels, R. L. G. M., \& Van Dongen, M. E. H.

Dynamic permeability: Reformulation of theory and new experimental 
and numerical data. Journal of Fluid Mechanics, 245(-1), 211. 10.1017/S0022112092000429

Sruoga, P., \& Rubinstein, N. (2007). Processes controlling porosity and permeability in volcanic reservoirs from the Austral and Neuquén basins, Argentina. American Association of Petroleum Geologists Bulletin, 91(1), 115-129. doi: $10.1306 / 08290605173$

Sruoga, P., Rubinstein, N., \& Hinterwimmer, G. (2004). Porosity and permeability in volcanic rocks: A case study on the Serie Tobifera, South Patagonia, Argentina. Journal of Volcanology and Geothermal Research, 132(1), 31-43. doi: 10.1016/S0377-0273(03)00419-0

Vidal, J., \& Genter, A. (2018). Overview of naturally permeable fractured reservoirs in the central and southern Upper Rhine Graben: Insights from geothermal wells. Geothermics, 74, 57-73. doi: 10.1016/j.geothermics.2018.02.003

White, J. E., Mihailova, N., \& Lyakhovitsky, F. (1975). Lowfrequency seismic waves in fluidsaturated layered rocks. The Journal of the Acoustical Society of America, 57(S1), S30-S30. doi: 10.1121/1.1995164

Wibberley, C. A., \& Shimamoto, T. (2003). Internal structure and permeability of major strike-slip fault zones: The Median Tectonic Line in Mie Prefecture, Southwest Japan. Journal of Structural Geology, 25(1), 59-78. doi: 10.1016/S0191-8141(02)00014-7

Yang, X., Cao, S., Guo, Q., Kang, Y., Yu, P., \& Hu, W. (2017). Frequencydependent amplitude versus offset variations in porous rocks with aligned fractures. $\quad$ Pure and Applied Geophysics, 174(3), 1043-1059. doi: 10.1007/ s00024-016-1423-8 